\title{
TORIC DEGENERATIONS AND BATYREV-BORISOV DUALITY
}

\author{
MARK GROSS
}

\section{INTRODUCTION.}

In [4], Bernd Siebert and I introduced the notion of a toric degeneration of CalabiYau varieties. The initial goal is to produce a method of constructing mirror pairs which combines the Strominger-Yau-Zaslow (differential geometric) approach to mirror symmetry and the older Batyrev-Borisov (algebro-geometric) approach to mirror symmetry. Our belief is that in doing so we will produce a new, much more powerful mechanism for constructing mirror pairs while gaining insight into the underlying structures of mirror symmetry.

Sketching the approach here, we showed in [4] how given a toric degeneration of CalabiYau varieties $\mathcal{X} \rightarrow \mathcal{S}$, one can associate to this degeneration a dual intersection complex $B$, which is an integral affine manifold with singularities. Here $\mathcal{S}$ is always a one-dimensional non-singular base. (See $\S 1$ of this paper for a quick review of this construction). In addition, if the family $\mathcal{X} \rightarrow \mathcal{S}$ is polarized with a relatively ample divisor, then $B$ comes along with a convex, piecewise linear multi-valued function $\varphi$. We then defined a notion of discrete Legendre transform, giving us a new affine manifold with singularities $\check{B}$ and a new function $\check{\varphi}$. We can interpret $\check{B}$ as the dual intersection complex of a new degeneration $\check{\mathcal{X}} \rightarrow \mathcal{S}$. This latter part of the program, at the time of this writing, is still not complete due to the question of existence of smoothings, although we do not anticipate major difficulties. In [4], we showed how to construct the degenerate fibre $\check{\mathcal{X}}_{0}$, along with a log structure on $\check{\mathcal{X}}_{0}$ which is crucial information for constructing a smoothing of $\check{\mathcal{X}}_{0}$. Once one knows the log scheme $\check{\mathcal{X}}_{0}$ can be smoothed, then the smoothing can be interpreted as an element of the mirror family.

Even without knowing whether the smoothing exists, this approach yields important information about the SYZ approach. Indeed, $B$ contains crucial information about the topology of the general fibre $\mathcal{X}_{t}$. As $B$ is an integral affine manifold with singularities, there is an open set $B_{0} \subseteq B$ which carries an integral affine structure, with $\Gamma:=B \backslash B_{0}$ a locally finite union of locally closed submanifolds of codimension at least two. Now we can define a topological space $X\left(B_{0}\right)$ as follows. Let $y_{1}, \ldots, y_{n}$ be local affine coordinates on $B_{0}$, and define a local system $\Lambda \subseteq \mathcal{T}_{B_{0}}$ to be the family of lattices generated by $\partial / \partial y_{1}, \ldots, \partial / \partial y_{n}$.

This work was partially supported by NSF grant 0204326 . 
Because of the integrality of the affine structure, this family of lattices is well-defined. We then define $X\left(B_{0}\right):=\mathcal{T}_{B_{0}} / \Lambda$; this is a torus bundle over $B_{0}$. (This manifold carries a complex structure also, see [3] for details.) In addition, given an element $\mathbf{B} \in H^{1}\left(B_{0}, \Lambda \otimes\right.$ $(\mathbb{R} / \mathbb{Z})$ ), one can twist the torus bundle by this element, obtaining a new torus bundle $X\left(B_{0}, \mathbf{B}\right) \rightarrow B_{0}$. In [5], we prove

Theorem 0.1. Let $\mathcal{X} \rightarrow \mathcal{S}$ be a toric degeneration, with dual intersection complex $B$. Then

(a) For $t \neq 0, \mathcal{X}_{t}$ is a topological compactification of $X\left(B_{0}, \mathbf{B}\right)$ for some $\mathbf{B} \in H^{1}\left(B_{0}, \Lambda \otimes\right.$ $(\mathbb{R} / \mathbb{Z}))$.

(b) If $B$ is simple (see [4], Definition 1.60), then there exists a $\mathbf{B} \in H^{1}\left(B, i_{*} \Lambda \otimes(\mathbb{R} / \mathbb{Z})\right.$ ) (with $i: B_{0} \hookrightarrow B$ the inclusion) and an algorithm for compactifying $X\left(B_{0}, \mathbf{B}\right) \rightarrow B_{0}$ to a torus fibration $X(B, \mathbf{B}) \rightarrow B$ so that $X(B, \mathbf{B})$ is homeomorphic to $\mathcal{X}_{t}$.

If we are given a pair of toric degenerations $\mathcal{X} \rightarrow \mathcal{S}$ and $\check{\mathcal{X}} \rightarrow \mathcal{S}$ whose dual intersection complexes are related by a discrete Legendre transform, then the torus bundles $X\left(B_{0}, \mathbf{B}\right) \rightarrow$ $B_{0}$ and $X\left(\check{B}_{0}, \check{\mathbf{B}}\right) \rightarrow \check{B}_{0}$ are in fact dual. Thus the above theorem gives us a very general tool for proving topological forms of the SYZ conjecture, so this approach is relevant even if one is not interested in producing new mirror pairs, or not yet able to do so.

This paper should be regarded as an extended example of our construction, though in fact it was part of the original motivation for the construction. We consider mirror pairs constructed by the Batyrev-Borisov construction. In this case, we already know of the existence of the mirror pairs, so we are checking several things. First, we wish to know that our construction gives the same answer as the Batyrev-Borisov construction. Second, we wish to know that a topological form of the SYZ construction holds for Batyrev-Borisov mirror pairs. This second fact follows from the first by the above theorem, so this paper is concerned with considering the first issue.

It is easy to construct examples of toric degenerations from the Batyrev-Borisov construction. For brevity in the introduction, let us consider only the toric hypersurface case. Let $M=\mathbb{Z}^{n}, M_{\mathbb{R}}=M \otimes \mathbb{R}, \Delta \subseteq M_{\mathbb{R}}$ be a reflexive polytope with 0 as unique interior integral point with reflexive dual $\Delta^{*}$. This defines a projective toric variety $\left(\mathbb{P}_{\Delta}, \mathcal{O}_{\mathbb{P}_{\Delta}}(1)\right)$. The toric variety $\mathbb{P}_{\Delta}$ is defined by a fan $\Sigma$ in $N_{\mathbb{R}}=M_{\mathbb{R}}{ }^{\vee}$ consisting of cones over faces of $\Delta^{*}$. Similarly, $\Delta^{*}$ defines a projective toric variety $\left(\mathbb{P}_{\Delta^{*}}, \mathcal{O}_{\mathbb{P}_{\Delta^{*}}}(1)\right)$ defined by a fan $\check{\Sigma}$ in $M_{\mathbb{R}}$. If we take a general section $s$ of $\mathcal{O}_{\mathbb{P}_{\Delta}}(1)$, and let $s_{0}$ be the section of $\mathcal{O}_{\mathbb{P}_{\Delta}}(1)$ corresponding to $0 \in \Delta$, then $t s+s_{0}=0$ defines a family $\mathcal{X}$ of Calabi-Yau hypersurfaces in $\mathcal{S} \times \mathbb{P}_{\Delta}$, where $t$ is a coordinate on $\mathcal{S}$. For $t=0$, we obtain a degenerate hypersurface consisting of the union of all toric divisors of $\mathbb{P}_{\Delta}$. In particular $\mathcal{X} \rightarrow \mathcal{S}$ is a toric degeneration. Now, it is very easy to see, and was shown in [4], that the dual intersection complex is just an affine structure (with singularities) on $\partial \Delta^{*}$, and that if we polarize the family with $\mathcal{O}_{\mathbb{P}_{\Delta}}(1)$, then 
the discrete Legendre transform immediately produces the dual intersection complex for a family of hypersurfaces arising similarly from the dual $\Delta^{*}$. However, this is too weak a statement for us, because these dual intersection complexes are probably never simple, so we can't apply part (b) of Theorem 0.1. Simplicity is essentially, as we shall see, an ampleness statement (Theorem 3.16). We need a more general form of degeneration to get simplicity. So we use a standard trick, already used in [17] in the context of the SYZ conjecture, and probably first introduced by Viro. Choose a height function $h: \Delta \cap M \rightarrow \mathbb{Z}$, and consider the degeneration

$$
s_{0}+\sum_{m \in \Delta \cap M} t^{h(m)} z^{m}=0 .
$$

With suitable restriction on $h$, when $t=0$ we again obtain just the equation $s_{0}=0$. However, the total family $\mathcal{X}$ defined by this equation is very singular, and toric techniques are required to resolve the singularities in order to obtain a toric degeneration.

More specifically, we will insist that $h$ be a restriction of an integral piecewise linear function on a subdivision of $\check{\Sigma}$ giving a partial crepant resolution $\check{\pi}: \tilde{\mathbb{P}}_{\Delta^{*}} \rightarrow \mathbb{P}_{\Delta^{*}}$ of $\mathbb{P}_{\Delta^{*}}$. Such a piecewise linear function determines a divisor $H_{\Delta^{*}}$ on $\tilde{\mathbb{P}}_{\Delta^{*}}$, and we insist that $H_{\Delta^{*}}$ should be ample and $H_{\Delta^{*}}+K_{\mathbb{P}_{\Delta^{*}}}$ be nef.

In this case, we show that the family defined by (1) is birationally equivalent to a toric degeneration $\mathcal{X} \rightarrow \mathcal{S}$, with general fibre being a Calabi-Yau hypersurface in $\tilde{\mathbb{P}}_{\Delta}$, where $\pi: \tilde{\mathbb{P}}_{\Delta} \rightarrow \mathbb{P}_{\Delta}$ is any partial crepant projective resolution. From this toric degeneration we obtain a dual intersection complex $B$. We show that if $\pi$ and $\check{\pi}$ are MPCP (maximal partial crepant projective) resolutions of $\mathbb{P}_{\Delta}$ and $\mathbb{P}_{\Delta^{*}}$, then $B$ is simple. Of course, in any event, we give an explicit combinatorial description of $B$ also.

The issue of duality is a bit more delicate. We show the following. If $H_{\Delta}$ is an ample polarization on $\tilde{\mathbb{P}}_{\Delta}$ induced by a piecewise linear function $\check{h}$, (with $H_{\Delta}+K_{\mathbb{P}_{\Delta}}$ nef for symmetry), we obtain a polarization on the generic fibre of $\mathcal{X} \rightarrow \mathcal{S}$. However, we can not always show that this polarization extends to $\mathcal{X}$. This seems to be a rather delicate combinatorial problem. However, if it does extend, then we can perform a discrete Legendre transform on $B$, obtaining a dual $\check{B}$, and we can show this is the dual intersection complex of a toric degeneration $\check{\mathcal{X}} \rightarrow \mathcal{S}$ obtained by interchanging $\Delta, h$ with $\Delta^{*}, \check{h}$. Thus the discrete Legendre transform provides a more sophisticated form of Batyrev (-Borisov) duality. The only difficulty is the existence of the polarization on $\mathcal{X}$. We obtain a weak existence result, showing that there exists an $m_{0}$ such that $m_{0} H_{\Delta}-n K_{\mathbb{P}_{\Delta}}$ extends to a polarization on $\mathcal{X}$ for $n$ sufficiently large. This is generally sufficient for our purposes, for if $H_{\Delta}$ is ample on $\tilde{\mathbb{P}}_{\Delta}$, so is $m_{0} H_{\Delta}-n K_{\mathbb{P}_{\Delta}}$.

Of course, all of this is done much more generally in the complete intersection case, which presents some new difficulties with the combinatorics; this paper would be much shorter if we only treated the hypersurface case. 
There has been important previous work covering the hypersurface case. W.-D. Ruan in [14] gave a combinatorial description of the SYZ fibration for hypersurfaces in toric varieties using his symplectic flow argument; he obtains essentially the same description that we do, though he doesn't use the language of affine manifolds. However, his proof of duality is hard to understand. Haase and Zharkov give an elegant explicit construction of the affine manifolds in the hypersurface case, in which duality is self-evident. They have independently generalized their construction to the complete intersection case [7].

The structure of this paper is as follows. As we try to be as self-contained as possible, so that the reader need only consult [4] from time to time, in $\S 1$ we review the notion of toric degeneration and dual intersection complex. However, notation of [4], $\S 1$ will be used throughout, so some familiarity with that paper will be useful. In $\S 2$, we consider the basic case of the Batyrev-Borisov construction, in which the polarizations used are the anti-canonical ones. While covering this case first may seem redundant, it is much simpler and much of the combinatorics is needed in the general case, which is covered in $\S 3$. In $\S 4$, we consider a worked example, one which gives a particularly nice example of a Strominger-Yau-Zaslow fibration which was recently studied by Kovalev. In this case, we see how the language of toric degenerations gives a much more flexible picture than the Batyrev-Borisov construction alone does.

I would like to thank Bernd Siebert, with whom the overall project of studying mirror symmetry via toric degenerations has been joint work, and Alexei Kovalev and Ilia Zharkov for useful conversations.

\section{REVIEW}

We recall the definition of toric degeneration from [4].

Definition 1.1. Let $R$ be a discrete valuation ring and $k$-algebra with algebraically closed residue class field $k$. A toric degeneration of Calabi-Yau varieties over $R$ is a proper normal algebraic space $\mathcal{X}$ flat over $\mathcal{S}:=\operatorname{Spec} R$ satisfying the following properties:

(1) The generic fibre $\mathcal{X}_{\eta}$ is an irreducible normal variety over $\eta$.

(2) $\mathcal{X}_{0}$ is reduced, and if $\nu: \tilde{\mathcal{X}}_{0} \rightarrow \mathcal{X}_{0}$ is the normalization, then $\tilde{\mathcal{X}}_{0}$ is a disjoint union of toric varieties, the conductor scheme $C \subseteq \tilde{\mathcal{X}}_{0}$ is reduced and the map $C \rightarrow \nu(C)$ is unramified and generically two-to-one. The square

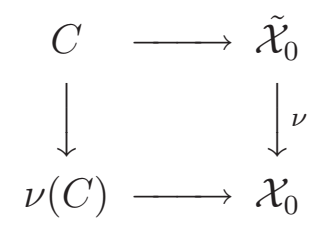

is cartesian and cocartesian.

(3) $\mathcal{X}$ (and hence $\mathcal{X}_{0}$ ) is Gorenstein, and the conductor locus $C$ restricted to each irreducible component of $\tilde{\mathcal{X}}_{0}$ is the union of all toric Weil divisors. 
(4) There exists a closed subset $Z \subseteq \mathcal{X}$ of relative codimension $\geq 2$ such that $Z$ satisfies the following properties: $Z$ does not contain the image under $\nu$ of any toric stratum of $\tilde{\mathcal{X}}_{0}$, and for any geometric point $\bar{x} \rightarrow \mathcal{X} \backslash Z$, there is an étale neighbourhood $U_{\bar{x}} \rightarrow \mathcal{X} \backslash Z$ of $\bar{x}$, an affine toric variety $Y_{\bar{x}}$, a regular function $f_{\bar{x}}$ on $Y_{\bar{x}}$ given by a monomial, a choice of uniformizing parameter of $R$ giving a map $k[\mathbb{N}] \rightarrow R$, and a commutative diagram

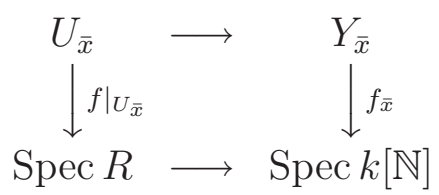

such that the induced map $U_{\bar{x}} \rightarrow \operatorname{Spec} R \times_{\operatorname{Spec} k[\mathbb{N}]} Y_{\bar{x}}$ is smooth. Furthermore, $f_{\bar{x}}$ vanishes on each toric divisor of $Y_{\bar{x}}$.

The slogan to remember here is that this definition roughly implies that $\mathcal{X}_{0}$ is a union of toric varieties meeting along toric strata, $\mathcal{X}_{0}$ has numerically trivial canonical class, and away from $Z, \mathcal{X} \rightarrow \mathcal{S}$ is described étale locally as a monomial map on an affine toric variety.

We recall how to construct the dual intersection complex of a toric degeneration $\mathcal{X} \rightarrow \mathcal{S}$. We will make the simplifying assumption in this review that all irreducible components of $\mathcal{X}_{0}$ are normal. We do this here as this will be the case with all examples constructed in this paper.

Write $\tilde{\mathcal{X}}_{0}=\coprod X_{i}$, with $\nu: \tilde{\mathcal{X}}_{0} \rightarrow \mathcal{X}_{0}$ the normalization. The set of strata of $\mathcal{X}_{0}$ is the set

$$
\operatorname{Strata}\left(\mathcal{X}_{0}\right):=\left\{\nu(S) \mid S \text { is a toric stratum of } X_{i} \text { for some } i\right\} .
$$

Associate to each strata of $\mathcal{X}_{0}$ a lattice polytope $P(S)$ as follows. If $S \in \operatorname{Strata}\left(\mathcal{X}_{0}\right)$, with generic point $\eta$, then by Condition (4) of Definition 1.1, there is a toric variety $Y_{\bar{\eta}}$ defined by a convex rational polyhedral cone $\sigma \subseteq M_{\mathbb{R}}$, where $M$ is a lattice and $M_{\mathbb{R}}=M \otimes \mathbb{R}$, so that $Y_{\bar{\eta}}=\operatorname{Spec} k\left[\sigma^{\vee} \cap N\right]$ with $N=\operatorname{Hom}(M, \mathbb{Z})$. In addition, there is an element $\rho \in \sigma^{\vee} \cap N$ inducing the map $Y_{\bar{\eta}} \rightarrow \operatorname{Spec} k[\mathbb{N}]$. Define

$$
P(S):=\{m \in \sigma \mid\langle\rho, m\rangle=1\} .
$$

One can show (see [4], §4) that $P(S)$ is a lattice polytope of dimension equal to the codimension of $S$ in $\mathcal{X}_{0}$.

Furthermore, if $S_{1} \subseteq S_{2}$ are two strata with corresponding cones $\sigma_{1}$ and $\sigma_{2}$, then toric geometry tells us that the faces of $\sigma_{1}$ are in one-to-one inclusion reversing correspondence with toric strata containing $S_{1}$. In particular, $\sigma_{2}$ is a face of $\sigma_{1}$ and $P\left(S_{2}\right)$ is naturally a face of $P\left(S_{1}\right)$. We can now construct the dual intersection complex $B$ of $\mathcal{X}$ as a union of polyhedra. Explicitly, if $S_{1}$ and $S_{2}$ are two zero-dimensional strata with $S_{3}$ the minimal stratum containing $S_{1}$ and $S_{2}$, then we identify $P\left(S_{1}\right)$ and $P\left(S_{2}\right)$ along the faces $P\left(S_{3}\right) \subseteq P\left(S_{1}\right)$ and $P\left(S_{3}\right) \subseteq P\left(S_{2}\right)$. In [4], Proposition 4.10, it was proved that $B$ is an 
$n$-dimensional manifold, where $n=\operatorname{dim} \mathcal{X}_{0}$. Furthermore, $B$ comes along with a polyhedral decomposition

$$
\mathscr{P}=\left\{P(S) \mid S \in \operatorname{Strata}\left(\mathcal{X}_{0}\right)\right\}
$$

Finally, $B$ can be given the structure of an integral affine manifold with singularities by specifying a fan structure at each vertex of $\mathscr{P}$. For each $v$ a vertex of $\mathscr{P}$, there is a corresponding irreducible component $X_{v}$ of $\mathcal{X}_{0}$, defined by a fan $\Sigma_{v} \subseteq \mathbb{R}^{n}$. Now each cone of $\Sigma_{v}$ corresponds to a toric stratum of $X_{v}$, and hence to an element of $\mathscr{P}$. This gives a combinatorial correspondence between cones of $\Sigma_{v}$ and cells of $\mathscr{P}$ containing $v$. This correspondence is a bijection in the case that all irreducible components of $\mathcal{X}_{0}$ are normal. Let $W_{v}$ be the union of interiors of all simplices in the first barycentric subdivision of $\mathscr{P}$ containing $v$ (the star of $v$ ). Then it is not difficult to see that one can define a map $\psi_{v}: W_{v} \rightarrow \mathbb{R}^{n}$ which is a homeomorphism onto its image, takes each cell of $\mathscr{P}$ containing $v$ into the corresponding cone of $\Sigma_{v}$, and is integral affine on the interiors of each maximal cell of $\mathscr{P}$ containing $v$. Together with the obvious charts

$$
\psi_{S}: \operatorname{Int}(P(S)) \hookrightarrow \mathbb{A}_{S}:=\left\{m \in M_{\mathbb{R}} \mid\langle\rho, m\rangle=1\right\}
$$

for $S$ a minimal stratum, we obtain an integral affine structure on $B_{0}:=B \backslash \Gamma$, where $\Gamma$ is the union of all simplices of $\operatorname{Bar}(\mathscr{P})$, the first barycentric subdivision of $\mathscr{P}$, not containing a vertex of $\mathscr{P}$ or intersecting the interior of a maximal cell of $\mathscr{P}$. This discriminant locus is usually much larger than necessary.

Recall that as $B_{0}$ carries an integral affine structure, we can define a local system $\Lambda \subseteq$ $\mathcal{T}_{B_{0}}$ which is the family of integral lattices locally generated by $\partial / \partial y_{1}, \ldots, \partial / \partial y_{n}$, where $y_{1}, \ldots, y_{n}$ are local integral affine coordinates. In [4], Proposition 1.27, it is shown that if the monodromy of $\Lambda$ about a simplex of $\Gamma$ is trivial, the affine structure of $B_{0}$ can be extended across this simplex. We shall use this latter fact in $\S 4$ to obtain a minimal discriminant locus in the example given there.

We will not review the construction of the discrete Legendre transform here, but rather refer the reader to either [3], $\S 4$ or [4], 1 1.4. Briefly, given an integral affine manifold with singularities $B$ with a polyhedral decomposition $\mathscr{P}$ and a multi-valued strictly convex piecewise linear function $\varphi$ on $B$, the discrete Legendre transform of the triple $(B, \mathscr{P}, \varphi)$ is a new triple $(\check{B}, \check{\mathscr{P}}, \check{\varphi})$ with $B=\check{B}$ topologically, but with different affine structures. The affine structures are dual in the sense that $\Lambda_{\breve{B}}=\operatorname{Hom}\left(\Lambda_{B}, \mathbb{Z}\right)$. Thus the torus bundles $X\left(B_{0}\right) \rightarrow B_{0}$ and $X\left(\check{B}_{0}\right) \rightarrow B_{0}$ are dual in the Strominger-Yau-Zaslow sense.

If $\mathcal{X} \rightarrow \mathcal{S}$ is a toric degeneration and $\mathcal{L}$ is a relatively ample line bundle on $\mathcal{X}$, we say we have a polarized toric degeneration. Restricting $\mathcal{L}$ to each irreducible component of $\mathcal{X}_{0}$ gives an ample line bundle on a toric variety, hence a strictly convex piecewise linear function 
on the corresponding fan. This gives rise to a multi-valued piecewise linear function $\varphi$ on the dual intersection complex $B$ of $\mathcal{X} \rightarrow \mathcal{S}$.

The discrete Legendre transform of $(B, \mathscr{P}, \varphi)$ in this case can be seen as the intersection complex of $\mathcal{X} \rightarrow \mathcal{S}$. This is obtained as follows. For every $k$-dimensional toric strata $S$ of $\mathcal{X}_{0},\left.\mathcal{L}\right|_{S}$, being an ample line bundle on $S$, has a Newton polytope, a $k$-dimensional lattice polytope $\check{P}(S) \subseteq \mathbb{R}^{k}$, well defined up to integral affine transformation. If $S_{1} \subseteq S_{2}$, then we can canonically identify $\check{P}\left(S_{1}\right)$ with a face of $\check{P}\left(S_{2}\right)$. Making these identifications we obtain $\check{B}, \check{\mathscr{P}}$. This is intuitively just a "real" drawing of the central fibre $\mathcal{X}_{0}$, obtained by dividing each component of $\mathcal{X}_{0}$ by its canonical real torus action.

To obtain an affine sturcture on $\check{B}$, we proceed as in the construction for $B$ : each maximal $\check{P}(S)$ has a canonical affine structure in its interior; for each zero-dimensional stratum $S$ we specify the fan structure at the vertex $\check{P}(S)$ to be the normal fan to $P(S)$. This yields $\check{B}$ as an affine manifold with singularities.

\section{The Batyrev-Borisov Construction: the Basic CASE}

We first recall the construction of Batyrev and Borisov. We will then demonstrate that the Batyrev-Borisov construction is a case of the discrete Legendre transform and gives rise to toric degenerations. This will be done first with the anti-canonical polarization, and we will cover the general polarization in the next section.

Definition 2.1. Let $M=\mathbb{Z}^{n}, N=\operatorname{Hom}(M, \mathbb{Z})$, and let $\Delta \subseteq M_{\mathbb{R}}$ be a reflexive polytope. Let $\Delta=\Delta_{1}+\cdots+\Delta_{r}, \Delta_{i} \subseteq M_{\mathbb{R}}$ be a Minkowski decomposition of $\Delta$ into lattice polytopes. Let $\Sigma \subseteq N_{\mathbb{R}}$ be the normal fan to $\Delta$ defining $\mathbb{P}_{\Delta}$, and $\varphi: N_{\mathbb{R}} \rightarrow \mathbb{R}$ be the integral (upper) convex $\Sigma$-piecewise linear function corresponding to the anti-canonical sheaf. If $\Sigma(1)=$ $\left\{e_{1}, \ldots, e_{m}\right\}$ (the set of integral generators of one-dimensional cones of $\left.\Sigma\right), \varphi\left(e_{i}\right)=1$. Then $\Delta=\Delta_{1}+\cdots+\Delta_{r}$ yields a decomposition $\varphi=\varphi_{1}+\cdots+\varphi_{r}$, where $\varphi_{i}$ defines the divisor corresponding to $\Delta_{i}$. Here

$$
\varphi_{i}\left(e_{j}\right)=-\inf \left\{\left\langle x, e_{j}\right\rangle \mid x \in \Delta_{i}\right\}
$$

and

$$
\Delta_{i}=\left\{x \in M_{\mathbb{R}} \mid\langle x, y\rangle \geq-\varphi_{i}(y) \quad \forall y \in N_{\mathbb{R}}\right\} .
$$

We say $\Delta=\Delta_{1}+\cdots+\Delta_{r}$ is a nef-partition if $\varphi_{i}\left(e_{j}\right) \in\{0,1\}$ for all $i, j$.

A nef-partition $\Delta=\Delta_{1}+\cdots+\Delta_{r}$ yields a decomposition $\mathcal{O}_{\mathbb{P}_{\Delta}}(1) \cong \mathcal{L}_{1} \otimes \cdots \otimes \mathcal{L}_{r}$, with $\mathcal{L}_{i}$ a semi-ample line bundle corresponding to $\Delta_{i}$. Recall (essentially [2], Proposition 4.15)

Proposition 2.2. Let $\Delta=\Delta_{1}+\cdots+\Delta_{r}$ be a nef partition, and $s$ be a general section of $\mathcal{L}_{1} \oplus \cdots \oplus \mathcal{L}_{r}$. Let $V \subseteq \mathbb{P}_{\Delta}$ be the zero-locus of $s$. If $V$ is non-empty and irreducible, then $V$ is an $n-r$ dimensional variety, $\omega_{V} \cong \mathcal{O}_{V}$, and $V$ has only canonical singularities. 
Definition 2.3. Given a nef partition, let $\nabla_{i}$ be the convex hull of $0 \in N_{\mathbb{R}}$ and all $e_{j}$ such that $\varphi_{i}\left(e_{j}\right)=1$. Set $\nabla=\nabla_{1}+\cdots+\nabla_{r}$.

Proposition 2.4. If $\nabla^{*}, \Delta^{*}$ are the duals of $\nabla$ and $\Delta$ respectively, then

$$
\begin{aligned}
& \nabla^{*}=\operatorname{Conv}\left\{\Delta_{1}, \ldots, \Delta_{r}\right\} \\
& \Delta^{*}=\operatorname{Conv}\left\{\nabla_{1}, \ldots, \nabla_{r}\right\} .
\end{aligned}
$$

Proof. [2], Theorem 4.10.

We also will use

\section{Lemma 2.5.}

$$
\left\langle\Delta_{j}, \nabla_{i}\right\rangle \geq-\delta_{j i}
$$

Proof. [1], Cor. 2.12.

Thus, to summarize: We are initially given data

$$
\begin{aligned}
\Delta & =\Delta_{1}+\cdots+\Delta_{r} \\
\nabla & =\nabla_{1}+\cdots+\nabla_{r} \\
\Delta^{*} & =\operatorname{Conv}\left\{\nabla_{1}, \ldots, \nabla_{r}\right\} \\
\nabla^{*} & =\operatorname{Conv}\left\{\Delta_{1}, \ldots, \Delta_{r}\right\} \\
\varphi & =\varphi_{1}+\cdots+\varphi_{r} \\
\check{\varphi} & =\check{\varphi}_{1}+\cdots+\check{\varphi}_{r}
\end{aligned}
$$

where $\varphi, \varphi_{i}$ are piecewise linear functions on the normal fan $\Sigma$ to $\Delta, \varphi$ representing $-K_{\mathbb{P}_{\Delta}}$, and $\check{\varphi}, \check{\varphi}_{i}$ are piecewise linear functions on the normal fan $\check{\Sigma}$ to $\nabla, \check{\varphi}$ representing $-K_{\mathbb{P}_{\nabla}}$. We have

$$
\begin{aligned}
\nabla_{i} & =\operatorname{Conv}\left(\left\{n \in \Sigma(1) \mid \varphi_{i}(n)=1\right\} \cup\{0\}\right) \\
\Delta_{i} & =\operatorname{Conv}\left(\left\{m \in \check{\Sigma}(1) \mid \check{\varphi}_{i}(m)=1\right\} \cup\{0\}\right) .
\end{aligned}
$$

We first work out the combinatorics of this duality from our point of view. In the hypersurface case, there is a straightforward dual relationship between faces of $\Delta$ and $\nabla=\Delta^{*}$. In the complete intersection case this is more delicate.

Definition 2.6. Let $\sigma^{*} \subset \Delta^{*}$ be a proper face. Let

$$
\sigma=\left\{m \in \Delta \mid\langle m, n\rangle=-1 \text { for all } n \in \sigma^{*}\right\}
$$

be the dual face of $\Delta$. Let

$$
\sigma_{i}^{*}=\left\{n \in \sigma^{*} \mid \varphi_{i}(n)=1\right\} \subseteq \nabla_{i}
$$

and set $\varrho=\sigma_{1}^{*}+\cdots+\sigma_{r}^{*} \subseteq \nabla$. (We take $\varrho$ to be empty if any $\sigma_{i}^{*}$ is empty). Let

$$
\iota^{*}=\left\{m \in \nabla^{*} \mid\langle m, n\rangle=-1 \text { for all } n \in \varrho\right\},
$$


and let

$$
\rho_{i}^{*}=\left\{m \in \varrho^{*} \mid \check{\varphi}_{i}(m)=1\right\} \subseteq \Delta_{i} .
$$

\section{Lemma 2.7.}

(a) Let $\sigma^{*} \subset \Delta^{*}$ be a proper face, and suppose $\Omega$ is non-empty. Then

$$
\begin{aligned}
\sigma^{*} & =\operatorname{Conv}\left(\sigma_{1}^{*}, \ldots, \sigma_{r}^{*}\right) \\
\varrho^{*} & =\operatorname{Conv}\left(\varrho_{1}^{*}, \ldots, \rho_{r}^{*}\right) \\
\sigma & =\rho_{1}^{*}+\cdots+\varrho_{r}^{*}
\end{aligned}
$$

and $\_$is a proper face of $\nabla$.

(b)

$$
\begin{aligned}
\varrho_{i}^{*} & =\left\{m \in \Delta_{i} \mid\langle m, n\rangle=-\varphi_{i}(n) \text { for all } n \in \sigma^{*}\right\} \\
\sigma_{i}^{*} & =\left\{n \in \nabla_{i} \mid\langle m, n\rangle=-\check{\varphi}_{i}(m) \text { for all } m \in \varrho^{*}\right\}
\end{aligned}
$$

Proof. (a) Clearly $\operatorname{Conv}\left(\sigma_{1}^{*}, \ldots, \sigma_{r}^{*}\right) \subseteq \sigma^{*}$. Conversely, let $n \in \sigma^{*}$ be a vertex. Then $\varphi_{i}(n)=1$ for some $i$, so $n \in \sigma_{i}^{*}$. Thus $\sigma^{*} \subseteq \operatorname{Conv}\left(\sigma_{1}^{*}, \ldots, \sigma_{r}^{*}\right)$. Similarly,

$$
\rho^{*}=\operatorname{Conv}\left(\rho_{1}^{*}, \ldots, \rho_{r}^{*}\right) .
$$

Next let $m \in \sigma$ be a vertex of $\sigma$. Since $\Delta=\Delta_{1}+\cdots+\Delta_{r}$, we can write $m=m_{1}+\cdots+m_{r}$ where $m_{i}$ is a vertex of $\Delta_{i}$. Suppose $m_{i}=0$. If $n \in \sigma_{i}^{*} \subseteq \sigma^{*}$ with $\varphi_{i}(n)=1$ (such exists by the assumption that $\rho$ is non-empty), then $-1=\langle m, n\rangle=\sum_{\substack{j=1 \\ i \neq j}}^{r}\left\langle m_{j}, n\right\rangle \geq 0$ by Lemma 2.5. Thus $m_{i} \neq 0$, so each $m_{i}$ is a non-zero vertex of $\Delta_{i}$, and $\check{\varphi}_{i}\left(m_{i}\right)=1$. Next, let $n \in \rho$, $n=n_{1}+\cdots+n_{r}$ with $n_{i} \in \sigma_{i}^{*} \subseteq \sigma^{*}$. Then

$$
-1=\left\langle m, n_{j}\right\rangle=\sum_{i=1}^{r}\left\langle m_{i}, n_{j}\right\rangle \geq-\sum_{i=1}^{r} \delta_{i j},
$$

so in fact $\left\langle m_{i}, n_{j}\right\rangle=-\delta_{i j}$. Thus $\left\langle m_{i}, n\right\rangle=-1$, so $m_{i} \in \varrho^{*}$, and hence $m_{i} \in \Omega_{i}^{*}$. We conclude $\sigma \subseteq \Omega_{1}^{*}+\ldots+\rho_{r}^{*}$. (Note in particular, we have shown $\Omega^{*}$ is non-empty if $\sigma$ is non-empty.)

Conversely, let $m=m_{1}+\cdots+m_{r} \in \rho_{1}^{*}+\cdots+\iota_{r}^{*}$. Then $m \in \Delta$. Let $n \in \sigma^{*}$ be a vertex, so $n \in \sigma_{i}^{*}$ for some $i$. Choose $n_{j} \in \sigma_{j}^{*}$ for $j \neq i$, and set $n_{i}=n$. Then as $m_{j} \in \rho^{*}$, $-1=\left\langle m_{j}, \sum_{k=1}^{r} n_{k}\right\rangle \geq-\sum_{i=1}^{r} \delta_{j k}$, so $\left\langle m_{j}, n_{k}\right\rangle=-\delta_{j k}$. Thus $\langle m, n\rangle=\left\langle m, n_{i}\right\rangle=-1$. Since this holds for every vertex $n$ of $\sigma^{*}, m \in \sigma$. Thus $\sigma=\rho_{1}^{*}+\cdots+\rho_{r}^{*}$.

Finally, as noted above, $\rho^{*}$ is non-empty, and hence $\rho$ is contained in a proper face of $\nabla$. Let $\rho^{\prime}$ denote the minimal face of $\nabla$ containing $\rho$, i.e. the face dual to $\rho^{*}$. Then interchanging $\Delta$ with $\nabla$ and $\nabla^{*}$ with $\Delta^{*}$, the statement $\sigma=\rho_{1}^{*}+\cdots+\rho_{r}^{*}$ proved above becomes $\varrho^{\prime}=\sigma_{1}^{*}+\cdots+\sigma_{r}^{*}$. Thus $\varrho=\varrho^{\prime}$.

(b) We just prove the first statement. If $m=m_{1}+\cdots+m_{r}$ with $m_{i} \in \rho_{i}^{*}, n_{j} \in \sigma_{j}^{*} \subseteq \sigma^{*}$, then we saw that $\left\langle m_{i}, n_{j}\right\rangle=-\delta_{i j}=-\varphi_{i}\left(n_{j}\right)$, from which it follows that $o_{i}^{*} \subseteq\{m \in$ 
$\left.\Delta_{i} \mid\langle m, n\rangle=-\varphi_{i}(n) \quad \forall n \in \sigma^{*}\right\}$. Conversely, let $m \in \Delta_{i}$ be a vertex with $\langle m, n\rangle=-\varphi_{i}(n)$ for all $n \in \sigma^{*}$. Since $\varrho \neq \emptyset$, this means $m \neq 0$, and $\check{\varphi}_{i}(m)=1$. Thus if $m \in \rho^{*}, m \in \rho_{i}^{*}$ as desired. Now if $n_{j} \in \sigma_{j}^{*} \subseteq \sigma^{*}, n=n_{1}+\cdots+n_{r} \in \rho$, and $\langle m, n\rangle=-\sum_{j} \varphi_{i}\left(n_{j}\right)=-1$, so $\langle m, n\rangle=-1$ for all $n \in \rho$, so $m \in \varrho^{*}$.

It is clear from this that given any one of $\sigma, \sigma^{*}, \varrho$ or $\rho^{*}$, we obtain the other three. We now state the following combinatorial duality, which follows immediately from the Lemma.

Corollary 2.8. Let

$$
\mathscr{R}_{\Delta^{*}}=\left\{\sigma^{*} \subset \Delta^{*} \mid \rho \neq \emptyset\right\}
$$

and

$$
\mathscr{R}_{\nabla^{*}}=\left\{\rho^{*} \subset \nabla^{*} \mid \sigma \neq \emptyset\right\}
$$

be subsets of the set of faces of $\Delta^{*}$ and $\nabla^{*}$ respectively. Let

$$
\mathscr{P}_{\nabla}=\left\{\rho \subset \nabla \mid \sigma^{*} \in \mathscr{R}_{\Delta^{*}}\right\}
$$

and

$$
\mathscr{P}_{\Delta}=\left\{\sigma \subset \Delta \mid \rho^{*} \in \mathscr{R}_{\nabla^{*}}\right\} .
$$

Then the maps $\mathscr{R}_{\Delta^{*}} \rightarrow \mathscr{P}_{\nabla}$ and $\mathscr{R}_{\nabla *} \rightarrow \mathscr{P}_{\Delta}$ given by $\sigma^{*} \mapsto \rho$ and $\rho^{*} \mapsto \sigma$ are order preserving bijections. The maps $\mathscr{R}_{\Delta^{*}} \rightarrow \mathscr{R}_{\nabla^{*}}$ given by $\sigma^{*} \mapsto \rho^{*}$ and $\mathscr{P}_{\nabla} \rightarrow \mathscr{P}_{\Delta}$ given by $\sigma \mapsto \varrho$ are order reversing bijections.

Proposition 2.9. If $\rho \in \mathscr{P}_{\nabla}$, any face of $\rho$ is also in $\mathscr{P}_{\nabla}$, and dually, for $\sigma \in \mathscr{P}_{\Delta}$. Also, if $\rho \in \mathscr{P}_{\nabla}$, then

$$
\begin{aligned}
\operatorname{dim} \varrho & =\operatorname{dim} \sigma^{*}-r+1 \\
\operatorname{dim} \sigma & =\operatorname{dim} \varrho^{*}-r+1 \\
\operatorname{dim} \varrho & =\left(\operatorname{dim} M_{\mathbb{R}}-r\right)-\operatorname{dim} \sigma
\end{aligned}
$$

Proof. Let $\perp$ be a face of $\varrho$. Then we obtain $\iota^{*} \supseteq \rho^{*}$, and hence $\perp_{i}^{*} \supseteq \rho_{i}^{*}$, and hence $\perp_{i}^{*}$ is non-empty for each $i$. Taking $\tau=\perp_{1}^{*}+\cdots+\perp_{r}^{*} \supseteq \sigma$, we obtain $\tau^{*} \subseteq \sigma^{*}$, and by the Lemma, $\perp=\tau_{1}^{*}+\cdots+\tau_{r}^{*}$. Thus $\perp \in \mathscr{P}_{\nabla}$.

It is enough to prove the first dimension statement. The second will be dually true and then $\operatorname{dim} \varrho=\operatorname{dim} \sigma^{*}-r+1=\left(\operatorname{dim} M_{\mathbb{R}}-\operatorname{dim} \sigma-1\right)-r+1$. To show the first dimension statement, we proceed as follows. First, if $\operatorname{dim} \rho=0$, then $\operatorname{dim} \sigma_{i}^{*}=0$ for each $i$, and $\sigma^{*}$ is a convex hull of vertices $v_{1}, \ldots, v_{r}$, with $\sigma_{i}^{*}=\left\{v_{i}\right\}$. Furthermore, since $\varphi_{i}\left(v_{j}\right)=\delta_{i j}$, $v_{1}, \ldots, v_{r}$ are linearly independent, so $\operatorname{dim} \sigma^{*}=r-1$.

Now let $\varrho \in \mathscr{P}_{\nabla}$ be arbitrary, with $\operatorname{dim} \rho=p$. Choose a vertex $\iota_{0}$ of $\rho$, and a vertex $\tau_{q}$ of $\sigma$, (here $q$ will be an index we will set in a moment equal to $\operatorname{dim} M_{\mathbb{R}}-r$ ). Then $\tau_{q}^{*}$ is a maximal face of $\Delta^{*}$ containing $\sigma^{*}$, and hence $\iota_{q}$ is a maximal element of $\mathscr{P}_{\nabla}$ containing $\iota$. On the other hand, arguing dually as above, since $\tau_{q}$ is a vertex, $\operatorname{dim} \perp_{q}^{*}=r-1$, so $\operatorname{dim} \perp_{q}=\operatorname{dim} M_{\mathbb{R}}-r$. Now take a strictly ascending chain of faces $\perp_{0} \subset \cdots \subset \perp_{q}$ 
with $\perp_{p}=\rho$, so that $\operatorname{dim} \perp_{i}=i$. We then obtain a strictly ascending chain of faces $\tau_{0}^{*} \subset \cdots \subset \tau_{q}^{*}$ with $\tau_{p}^{*}=\sigma^{*}$. Since $\operatorname{dim} \tau_{0}^{*}=r-1$ and $\operatorname{dim} \tau_{q}^{*}=\operatorname{dim} M_{\mathbb{R}}-1$, it follows that $\operatorname{dim} \tau_{i}^{*}=r-1+i$ and $\operatorname{dim} \sigma^{*}=r-1+\operatorname{dim} \rho$, as desired.

Definition 2.10. Let

$$
\begin{aligned}
& B_{\nabla}=\bigcup_{\rho \in \mathscr{P}_{\nabla}} \rho \subseteq \nabla \\
& B_{\Delta}=\bigcup_{\sigma \in \mathscr{P}_{\Delta}} \sigma \subseteq \Delta
\end{aligned}
$$

so that $\mathscr{P}_{\nabla}$ and $\mathscr{P}_{\Delta}$ give polyhedral decompositions of $B_{\nabla}$ and $B_{\Delta}$ respectively, with first barycentric subdivisions $\operatorname{Bar}\left(\mathscr{P}_{\nabla}\right)$ and $\operatorname{Bar}\left(\mathscr{P}_{\Delta}\right)$. We define a discriminant locus of $B_{\nabla}$, $\Gamma_{\nabla}$, as the union of all simplices of $\operatorname{Bar}\left(\mathscr{P}_{\nabla}\right)$ not containing a vertex of $\mathscr{P}_{\nabla}$ or intersecting the interior of a maximal cell of $\mathscr{P}_{\nabla}$. We do the same dually to define $\Gamma_{\Delta} \subseteq B_{\Delta}$.

We define an affine structure on $B_{\nabla} \backslash \Gamma_{\nabla}$ as follows. For every maximal cell $\rho$ of $B_{\nabla}$, let $\mathbb{A}_{\rho}$ be the affine subspace of $N_{\mathbb{R}}$ spanned by $\rho$. Then we define an affine chart on $\operatorname{Int}(\rho)$ by $\psi_{\varrho}: \operatorname{Int}(\rho) \hookrightarrow \mathbb{A}_{\varrho}$. Secondly, for $v \in \mathscr{P}_{\nabla}$ a vertex, we have

$$
v=\sum_{i=1}^{r} v_{i}
$$

for some $v_{i} \in \Sigma(1)$. Let $W_{v}$ be the (open) star of $v$ in $\operatorname{Bar}\left(\mathscr{P}_{\nabla}\right)$, i.e. the union of interiors of all simplices of $\operatorname{Bar}\left(\mathscr{P}_{\nabla}\right)$ containing $v$. We then define an affine chart

$$
\psi_{v}: W_{v} \rightarrow N_{\mathbb{R}} / \operatorname{Span}\left(v_{1}, \ldots, v_{r}\right)
$$

simply via projection.

Similarly we define an affine structure on $B_{\Delta} \backslash \Gamma_{\Delta}$.

If $\sigma \subseteq \mathbb{R}^{n}$, we always write

$$
C(\sigma)=\{r m \mid r \geq 0, m \in \sigma\}
$$

for the cone over $\sigma$.

\section{Lemma 2.11.}

(a) Let $\sigma^{*} \in \mathscr{R}_{\Delta^{*}}$. Let $m_{1}, \ldots, m_{r} \in M_{\mathbb{R}} / C\left(\sigma^{*}\right)^{\perp}$ be such that $\left\langle m_{i}, n\right\rangle=\varphi_{i}(n)$ for all $n \in \sigma^{*}$, and write

$$
V_{\sigma}=\operatorname{Span}\left\{m_{i}-m_{j} \mid 1 \leq i, j \leq r\right\} \subseteq M_{\mathbb{R}} / C\left(\sigma^{*}\right)^{\perp} .
$$

This gives a subspace $V_{\sigma}^{\perp} \subseteq \mathbb{R} C\left(\sigma^{*}\right) \subseteq N_{\mathbb{R}}$. Then

$$
C(\rho)=V_{\sigma}^{\perp} \cap C\left(\sigma^{*}\right) .
$$


(b) If $\tau^{*} \subseteq \sigma^{*}, \tau^{*}, \sigma^{*} \in \mathscr{R}_{\Delta^{*}}$, then there is an isomorphism $\mathbb{R} C(\rho) / \mathbb{R} C(\perp) \cong \mathbb{R} C\left(\sigma^{*}\right) / \mathbb{R} C\left(\tau^{*}\right)$ inducing an isomorphism of cones

$$
(C(\rho)+\mathbb{R} C(\perp)) / \mathbb{R} C(\perp) \cong\left(C\left(\sigma^{*}\right)+\mathbb{R} C\left(\tau^{*}\right)\right) / \mathbb{R} C\left(\tau^{*}\right) .
$$

Proof. (a) Since $\left\langle m_{i}, \sigma_{j}^{*}\right\rangle=\delta_{i j}, m_{i}=m_{j}$ on $\_=\sigma_{1}^{*}+\cdots+\sigma_{r}^{*}$. Thus $\_\subseteq V_{\sigma}^{\perp} \cap C\left(\sigma^{*}\right)$. Conversely, if $n \in V_{\sigma}^{\perp} \cap C\left(\sigma^{*}\right)$, then either $\left\langle m_{i}, n\right\rangle=0$ for all $i$, in which case $n=0$, or we can multiply $n$ by a positive real number so that we can assume $\left\langle m_{i}, n\right\rangle=1$ for all $i$. We wish to prove that after doing so, $n \in \rho$. For this, it is enough to show $\langle m, n\rangle=-1$ if $m \in \varrho^{*}$ and $\langle m, n\rangle \geq-1$ if $m \in \nabla^{*}$. But $\iota^{*}=\operatorname{Conv}\left(\rho_{1}^{*}, \ldots, \rho_{r}^{*}\right)$, and if $m \in \Omega_{i}^{*}$, then $m$ defines the function $-\varphi_{i}$ on $C\left(\sigma^{*}\right)$ by Lemma 2.7, (b), and so $\langle m, n\rangle=-1$. Thus if $m \in \rho^{*},\langle m, n\rangle=-1$. If $m \in \Delta_{i}$, then $\langle m, n\rangle \geq-\varphi_{i}(n)=-1$, so if $m \in \nabla^{*}=\operatorname{Conv}\left(\Delta_{1}, \ldots, \Delta_{r}\right),\langle m, n\rangle \geq-1$. Thus $n \in \rho$ as desired.

(b) Note that $\mathbb{R} C(\rho)=V_{\sigma}^{\perp}$ : certainly by (a) $\mathbb{R} C(\rho) \subseteq V_{\sigma}^{\perp}$, and $\operatorname{dim} C(\rho)=$ $\operatorname{dim} C\left(\sigma^{*}\right)-r+1$ by Proposition 2.9 while $\operatorname{dim} V_{\sigma}^{\perp}=\operatorname{dim} \mathbb{R} C\left(\sigma^{*}\right)-(r-1)=\operatorname{dim} C(\rho)$. Thus the two vector spaces coincide. Now we have a map $V_{\sigma}^{\perp} / V_{\tau}^{\perp} \rightarrow \mathbb{R} C\left(\sigma^{*}\right) / \mathbb{R} C\left(\tau^{*}\right)$, which is injective since $V_{\tau}^{\perp}=V_{\sigma}^{\perp} \cap \mathbb{R} C\left(\tau^{*}\right)$, and is surjective, as can be easily seen by calculating dimensions. Since $C(\rho) \subseteq C\left(\sigma^{*}\right)$, clearly we then get an inclusion of cones $(C(\rho)+\mathbb{R} C(\iota)) / \mathbb{R} C(\lrcorner) \subseteq\left(C\left(\sigma^{*}\right)+\mathbb{R} C\left(\tau^{*}\right)\right) / \mathbb{R} C\left(\tau^{*}\right)$. On the other hand, if $n \in C\left(\sigma^{*}\right)$, there exists $n^{\prime} \in \mathbb{R} C\left(\tau^{*}\right)$ such that $n+n^{\prime} \in \mathbb{R} C(\rho)$. Now, if there is an $n^{\prime \prime} \in C(\perp)$ such that $n^{\prime \prime} \notin \partial C\left(\tau^{*}\right)$, then for some sufficiently large $r \in \mathbb{R}, n^{\prime}+r n^{\prime \prime} \in C\left(\tau^{*}\right)$. However, it is easy to see that $C(\perp) \nsubseteq \partial C\left(\tau^{*}\right)$, hence we have $n+n^{\prime}+r n^{\prime \prime} \in C(\rho)$. Thus the inclusion of cones is a surjection.

Proposition 2.12. The charts $\psi_{v}$ and $\psi_{\sigma}$ define an integral affine structure on $B_{\nabla} \backslash \Gamma_{\nabla}$, making $B_{\nabla}$ an integral affine manifold with singularities of dimension $\operatorname{dim} M_{\mathbb{R}}-r$.

Proof. The open sets of the form $\operatorname{Int}(\sigma)$ and $W_{v}$ clearly cover $B_{\nabla} \backslash \Gamma_{\nabla}$, so to show we obtain an integral affine structure, we need to show $\psi_{v}$ is a $C^{0}$ immersion and that the transition maps are integral affine. Let $v=\sum_{j=1}^{r} v_{j}$ be a vertex of $\mathscr{P}_{\nabla}$ and let $\rho$ be any maximal cell containing $v$ and $\mathbb{A}_{\rho}$ the affine space spanned by it. The functions $\varphi_{i}$ are linear on the cone over $\sigma^{*}$, and hence are induced by linearly independent elements $m_{i} \in M$ on $\sigma^{*}$. The space $\mathbb{A}_{\rho}$ is then given by

$$
\mathbb{A}_{\varrho}=\left\{x \in N_{\mathbb{R}} \mid\left\langle m_{i}, x\right\rangle=1 \text { for } i=1, \ldots, r\right\},
$$

as follows from Lemma 2.11.

Also

$$
\left\langle m_{i}, \sum_{j=1}^{r} a_{j} v_{j}\right\rangle=a_{i},
$$

so if $\sum_{j=1}^{r} a_{j} v_{j} \in \mathbb{A}_{\rho}$, we have $a_{j}=1$, for all $j$. Thus

$$
\mathbb{A}_{\rho} \cap \operatorname{Span}\left(v_{1}, \ldots, v_{r}\right)=\{v\} .
$$


In particular, the projection

$$
\mathbb{A}_{\varrho} \rightarrow N_{\mathbb{R}} / \operatorname{Span}\left(v_{1}, \ldots, v_{r}\right)
$$

is an affine isomorphism preserving integral structures, i.e. mapping $\mathbb{A} \rho \cap N$ isomorphically to $N /\left(\operatorname{Span}\left(v_{1}, \ldots, v_{r}\right) \cap N\right)$. Thus the transition maps are integral affine.

We then have to show that $\psi_{v}$ is 1-1. First let's look at the tangent wedge to $\psi_{v}(0)$ at $0=\psi_{v}(v)$. Let $\tau^{*}=\operatorname{Conv}\left(v_{1}, \ldots, v_{r}\right)$. Then the tangent cone to $\psi_{v}(\rho)$ is precisely the cone $(C(\rho)+\mathbb{R} v) / \mathbb{R} v$, equal to $\left(C\left(\sigma^{*}\right)+\mathbb{R} C\left(\tau^{*}\right)\right) / \mathbb{R} C\left(\tau^{*}\right)$ of the fan $\Sigma\left(C\left(\tau^{*}\right)\right)$ by Lemma 2.11, (b), (see [4], Definition 1.37 for notation). Since the maximal facets of $\mathscr{P}_{\nabla}$ containing $v$ are in one-to-one correspondence with maximal cones of $\Sigma$ containing $C\left(\tau^{*}\right)$, we see that the tangent wedges to all the $\psi_{v}(\rho)$ 's fit together to form the fan $\Sigma\left(C\left(\tau^{*}\right)\right)$. Thus in particular $\psi_{v}$ is a $\left(C^{0}\right)$ immersion.

The computation of monodromy can be done via a calculation of the sort carried out by Ruan in [14] or Haase and Zharkov in [6].

Proposition 2.13. Let $v$ and $v^{\prime}$ be two vertices of $\mathscr{P}_{\nabla}$ and let $\Omega$ and $\Omega^{\prime}$ be two maximal faces of $\mathscr{P}$ containing $v$ and $v^{\prime}$. Let $\gamma$ be a simple loop based at $v$, passing successively into $\operatorname{Int}(\rho)$, through $v^{\prime}$, into $\operatorname{Int}\left(\rho^{\prime}\right)$ and back to $v$. Write $v=v_{1}+\cdots+v_{r}$ and $v^{\prime}=v_{1}^{\prime}+\cdots+v_{r}^{\prime}$, for $v_{j}, v_{j}^{\prime} \in \Sigma(1)$, and let $m_{1}, \ldots, m_{r}, m_{1}^{\prime}, \ldots, m_{r}^{\prime} \in M$ define the functions $-\varphi_{i}$ on $\sigma$ and $\sigma^{\prime}$ respectively. Identifying $\Lambda_{v}$ with $N /\left(N \cap \operatorname{Span}\left(v_{1}, \ldots, v_{r}\right)\right)$, parallel transport $T_{\gamma}: \Lambda_{v} \rightarrow \Lambda_{v}$ around $\gamma$ is given by

$$
T_{\gamma}(n)=n+\sum_{j=1}^{r}\left\langle m_{j}^{\prime}-m_{j}, n\right\rangle\left(v_{j}^{\prime}-v_{j}\right)
$$

Proof. We can identify the tangent space to a point in $\operatorname{Int}(\rho)$ with $\operatorname{Span}\left(m_{1}, \ldots, m_{r}\right)^{\perp}$ and the tangent space to a point in $\operatorname{Int}\left(\Omega^{\prime}\right)$ with $\operatorname{Span}\left(m_{1}^{\prime}, \ldots, m_{r}^{\prime}\right)^{\perp}$. Then $T_{\gamma}$ is given by the following chain of identifications in a clockwise order:

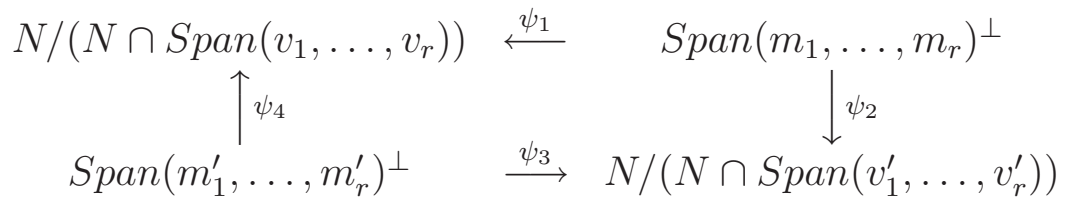

where each arrow is given by projection. Noting that

$$
\left\langle m_{i}, v_{j}\right\rangle=\left\langle m_{i}^{\prime}, v_{j}\right\rangle=\left\langle m_{i}, v_{j}^{\prime}\right\rangle=\left\langle m_{i}^{\prime}, v_{j}^{\prime}\right\rangle=-\delta_{i j}
$$


we get for $n \in N /\left(N \cap \operatorname{Span}\left(v_{1}, \ldots, v_{r}\right)\right)$ that

$$
\begin{aligned}
T_{\gamma}(n) & =\psi_{4}\left(\psi_{3}^{-1}\left(\psi_{2}\left(\psi_{1}^{-1}(n)\right)\right)\right) \\
& =\psi_{4}\left(\psi_{3}^{-1}\left(\psi_{2}\left(n+\sum_{j=1}^{r}\left\langle m_{j}, n\right\rangle v_{j}\right)\right)\right) \\
& =\psi_{4}\left(n+\sum_{j=1}^{r}\left\langle m_{j}, n\right\rangle v_{j}+\sum_{k=1}^{r}\left\langle m_{k}^{\prime}, n+\sum_{j=1}^{r}\left\langle m_{j}, n\right\rangle v_{j}\right\rangle v_{k}^{\prime}\right) \\
& =n+\sum_{k=1}^{r}\left(\left\langle m_{k}^{\prime}, n\right\rangle-\left\langle m_{k}, n\right\rangle\right) v_{k}^{\prime} \bmod \operatorname{Span}\left(v_{1}, \ldots, v_{r}\right) \\
& =n+\sum_{j=1}^{r}\left\langle m_{j}^{\prime}-m_{j}\right\rangle\left(v_{j}^{\prime}-v_{j}\right) \bmod \operatorname{Span}\left(v_{1}, \ldots, v_{r}\right)
\end{aligned}
$$

as desired.

Definition 2.14. It follows from Lemma 2.11 that if $\perp \in \mathscr{P}_{\nabla}$, then the fan $\Sigma_{\perp}$ (see [4], Definition 1.35) coincides with the quotient fan $\Sigma\left(C\left(\tau^{*}\right)\right)$. Define $\varphi \perp$ on the fan $\Sigma\left(C\left(\tau^{*}\right)\right)$ by choosing $m \in M$ such that $\langle m, n\rangle=\varphi(n)$ for all $n \in \tau^{*}$, so that $\varphi-m$ descends to a piecewise linear function $\varphi_{\perp}$ on the fan $\Sigma\left(C\left(\tau^{*}\right)\right)=\Sigma_{\perp}$; the function $\varphi_{\perp}$ is well-defined up to a linear function. Such a function also pulls back to a function on $W \perp \subseteq B_{\nabla}$ under the projection $W_{\perp} \rightarrow N_{\mathbb{R}} / \mathbb{R} C\left(\tau^{*}\right)$, where $W_{\perp}$ is the union of interiors of all simplices of $\operatorname{Bar}\left(\mathscr{P}_{\nabla}\right)$ containing the barycentre of $\perp$. Then $\varphi_{\nabla}=\left\{\left(W_{\perp}, \varphi_{\perp}\right)\right\}$ defines a multi-valued piecewise linear function on $B_{\nabla}$. ([4], Definition 1.42.) Similarly, we obtain $\check{\varphi}_{\Delta}$ on $B_{\Delta}$ from $\check{\varphi}$.

Theorem 2.15. $\left(B_{\Delta}, \mathscr{P}_{\Delta}, \check{\varphi}_{\Delta}\right)$ is the discrete Legendre transform of $\left(B_{\nabla}, \mathscr{P}_{\nabla}, \varphi_{\nabla}\right)$.

Proof. We already have a one-to-one inclusion reversing correspondence between $\mathscr{P}_{\nabla}$ and $\mathscr{P}_{\Delta}$ by Corollary 2.8. To show the result, we need to show (1) for $\varrho \in \mathscr{P}_{\nabla}$ a vertex, $\sigma$ is the Newton polytope of $\varphi_{\rho}$ on the fan $\Sigma_{\rho}$, and dually (2) for $\sigma \in \mathscr{P}_{\Delta}$ a vertex, $\rho$ is the Newton polytope of $\check{\varphi}_{\sigma}$ on the fan $\Sigma_{\sigma}$.

For (1), $\Sigma_{\varrho}=\Sigma\left(C\left(\sigma^{*}\right)\right)$ by Lemma 2.11, and as $\Delta$ is the Newton polytope of the function $\varphi$ on the fan $\Sigma$, the face $\sigma$ is the Newton polytope of the function $\varphi_{\rho}$ on $\Sigma_{\sigma}$. For (2) the dual argument works.

We now relate these combinatorial constructions to toric degenerations. Each line bundle $\mathcal{L}_{i}$ comes with a canonical section $s_{i}^{0}$ given by $0 \in \Delta_{i}$, whose zero locus is the toric divisor defined by $\varphi_{i}$. Let $s_{0}:=\left(s_{1}^{0}, \ldots, s_{r}^{0}\right) \in \Gamma\left(\mathcal{L}_{1} \oplus \cdots \oplus \mathcal{L}_{r}\right)$, and let $s=\left(s_{1}, \ldots, s_{r}\right)$ be a general section of $\mathcal{L}_{1} \oplus \cdots \oplus \mathcal{L}_{r}$. Then

$$
t s+s_{0}=0
$$


defines a family $\mathcal{X}$ in $\mathbb{P}_{\Delta} \times \mathcal{S}$ with $\mathcal{S}=$ Spec $R$, and $R$ is a discrete valuation ring with uniformizing parameter $t$. The projection $f: \mathcal{X} \rightarrow \mathcal{S}$ gives our family.

Let us assume that the zero locus of $s$ is non-empty and irreducible, and hence by Proposition 2.2, if $s$ is chosen generically, this zero locus has only canonical singularities.

Proposition 2.16. $f: \mathcal{X} \rightarrow \mathcal{S}$ is a toric degeneration of Calabi-Yau varieties, with dual intersection complex $B_{\nabla}, \mathscr{P}_{\nabla}$.

Proof. We first check that $\operatorname{dim} \mathcal{X}_{0}=n-r$. Consider the irreducible components of $\mathcal{X}_{0}$. The divisor given by $s_{i}^{0}=0$ is $\sum \varphi_{i}\left(e_{j}\right) D_{j}$, where $D_{j}$ is the (Weil) divisor of $\mathbb{P}_{\Delta}$ corresponding to the ray $e_{j}$ of $\Sigma$, and $\Sigma(1)=\left\{e_{1}, \ldots, e_{m}\right\}$. Then an irreducible component of $s_{0}=0$ is $\bigcap_{j=1}^{r} D_{i_{j}}$, for some $i_{1}, \ldots, i_{r}$ where $\varphi_{j}\left(e_{i_{j}}\right)=1$. This intersection is either empty, or is a toric stratum of $\mathbb{P}_{\Delta}$ defined by the smallest cone $C\left(\sigma^{*}\right)$ of $\Sigma$ containing $e_{i_{1}}, \ldots, e_{i_{r}}$. Suppose $\operatorname{dim} C\left(\sigma^{*}\right)<r$. Then $e_{i_{1}}, \ldots, e_{i_{r}}$ are linearly dependent. However, each $\varphi_{j}$ is linear on $C\left(\sigma^{*}\right)$, and since $\varphi_{j}\left(e_{i_{k}}\right)=\delta_{j k}, e_{i_{1}}, \ldots, e_{i_{r}}$ must be linearly independent. So $\operatorname{dim} C\left(\sigma^{*}\right) \geq r$, and $\mathcal{X}_{0}$ has no irreducible component of dimension $>n-r$, as desired.

Now $\mathcal{X}_{\eta}$ is an irreducible normal variety with canonical singularities of dimension $n-r$ by Proposition 2.2, and $\operatorname{dim} \mathcal{X}=n-r+1$. However, $\mathbb{P}_{\Delta} \times \mathcal{S}$ is Gorenstein and since $\mathcal{X}$ is defined by $r$ equations, $\mathcal{X}$ can have no components (embedded or otherwise) of dimension $\leq n-r$ by the unmixedness theorem [12], Thereom 32. Thus $\mathcal{X}$ is irreducible, as $\mathcal{X}_{0}$ has no component of dimension greater than $n-r$. Finally, as $\mathcal{X}$ is a complete intersection in $\mathbb{P}_{\Delta} \times \mathcal{S}$, it is also Gorenstein. Thus $f: \mathcal{X} \rightarrow \mathcal{S}$ is proper and flat over $S$, and (1) of Definition 1.1 holds.

Next we define

$$
Z_{0}=\operatorname{Sing}\left(\mathcal{X}_{0}\right) \cap \bigcup_{i=1}^{r}\left\{s_{i}=0\right\} .
$$

Since the $\mathcal{L}_{i}$ are semi-ample, and $s$ is general, $s_{i}$ does not vanish identically on any toric stratum.

Let $\bar{x} \rightarrow \mathcal{X}_{0}$ be a geometric point where none of the $s_{i}$ 's vanish, with image a point $x \in \mathcal{X}_{0}$. Then the closure of the torus orbit of $x$ in $\mathbb{P}_{\Delta}$ is a toric stratum corresponding to some cone $C\left(\sigma^{*}\right)$ of $\Sigma$. This cone $C\left(\sigma^{*}\right)$ induces an affine open subset

$$
U_{\sigma}=\operatorname{Spec} k\left[C\left(\sigma^{*}\right)^{\vee} \cap M\right]
$$

of $\mathbb{P}_{\Delta}$, with $x \in U_{\sigma}$. If $m \in M$, we denote by $z^{m}$ the corresponding monomial. On $U_{\sigma}, \mathcal{L}_{i}$ is trivial, and we can view the sections $s_{i}$ and $s_{i}^{0}$ as regular functions on $U_{\sigma}$. Explicitly, if $m_{i} \in M$ defines the function $\varphi_{i}$ on the cone $C\left(\sigma^{*}\right)$, then $m_{i} \in C\left(\sigma^{*}\right)^{\vee} \cap M$ can be taken to represent the sections $s_{i}^{0}$, up to an invertible function on $U_{\sigma}$. We can then write the equations of $\mathcal{X}$ in $U_{\sigma} \times \mathcal{S}$ as

$$
t f_{i}-z^{m_{i}}=0, \quad i=1, \ldots, r
$$


with $f_{i}$ an invertible function times $s_{i}$. Since all the $f_{i}$ 's are non-zero at $x$, a neighbourhood of $x$ is locally étale equivalent to the subscheme of $U_{\sigma} \times \mathcal{S}$ defined by

$$
t-z^{m_{i}}=0 \quad i=1, \ldots, r .
$$

To see this note that if we choose a basis $e_{1}, \ldots, e_{n}$ of $M$, and $g_{1}, \ldots, g_{n}$ are functions on $U_{\sigma} \times \mathcal{S}$ invertible on a neighbourhood $V$ of $x$, then we obtain in some neighbourhood of $x$ a local isomorphism $V \rightarrow U_{\sigma} \times \mathcal{S}$ via $z^{e_{i}} \mapsto g_{i} z^{e_{i}}$. After taking roots of the functions $f_{i}$, i.e. after passing to an étale cover, we can find $g_{i}$ 's such that $z^{m_{i}} \mapsto f_{i} z^{m_{i}}$. Under this map the equation $t f_{i}-z^{m_{i}}=0$ is taken to $t-z^{m_{i}}=0$. Note this is locally the same thing as the subscheme of $U_{\sigma}$ defined by

$$
z^{m_{i}}=z^{m_{j}}, \quad 1 \leq i<j \leq r .
$$

This is a toric subvariety of $U_{\sigma}$, defined by the following cone. Let $V_{\sigma} \subseteq M_{\mathbb{R}}$ be the subspace spanned by $\left\{m_{i}-m_{j} \mid 1 \leq i<j \leq r\right\}$. Then by Lemma 2.11,

$$
C(\rho)=C\left(\sigma^{*}\right) \cap V_{\sigma}^{\perp} \subseteq V_{\sigma}^{\perp}
$$

defines an $n-r+1$ dimensional toric variety $U_{\rho}$ with $C(\rho)^{\vee} \subseteq\left(V_{\sigma}^{\perp}\right)^{\vee}=M_{\mathbb{R}} / V_{\sigma}$, so that the monomials $z^{m_{i}}$ all agree on $U_{\rho}$. Then the inclusion $V_{\sigma}^{\perp} \subseteq N_{\mathbb{R}}$ induces a closed embedding of $U_{\rho}$ in $U_{\sigma}$, with image defined by the equations $z^{m_{i}}=z^{m_{j}}$, and the map $U_{\varrho} \rightarrow$ Spec $k[t]$ is defined by any of the monomials $z^{m_{i}}$. Thus there is an étale neighbourhood $U_{\bar{x}}$ of $x$ in $\mathcal{X}$ and a diagram as desired

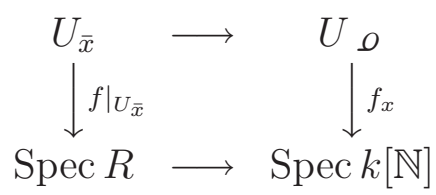

where $f_{x}$ is given by any of the $z^{m_{i}}$. Now since $\left\langle m_{i}, n\right\rangle=1$ for any $n \in \rho, f_{x}$ vanishes precisely once on each toric divisor of $U_{\varrho}$. This demonstrates that at $\bar{x}$ the condition of (4) of Definition 1.1 holds, and that $\mathcal{X}_{0}$ is reduced. Note that if $x \notin \operatorname{Sing}\left(\mathcal{X}_{0}\right)$, $f$ is then smooth at $x$ and the condition of (4) holds vacuously. In particular, $\mathcal{X}$ is regular in codimension one and hence normal. We can then take $Z=Z_{0} \cup \overline{\operatorname{Sing}\left(\mathcal{X}_{\eta}\right)}$ where $\mathcal{X}_{\eta}$ is the generic fibre of $f$. From the above it is clear that $Z$ satisfies the conditions of (4).

The rest of condition (2) of Definition 1.1 follows, and condition (3) is easily checked.

That $B_{\nabla}, \mathscr{P}_{\nabla}$ is the dual intersection complex of $f: \mathcal{X} \rightarrow \mathcal{S}$ now follows immediately from the construction described in $\S 1$ : If $\sigma^{*} \in \mathscr{R}_{\Delta^{*}}$ is maximal corresponding to a zerodimensional stratum of $\mathcal{X}_{0}$, then by above the corresponding maximal cell in the dual intersection complex is $\rho$. If $\sigma^{*} \in \mathscr{R}_{\Delta^{*}}$ corresponds to an irreducible component of $\mathcal{X}_{0}$, then this component is defined by the fan $\Sigma\left(C\left(\sigma^{*}\right)\right)=\Sigma \Omega_{\varrho}$, as desired.

Remark 2.17. $B_{\nabla}$ and $B_{\Delta}$ need not be topological spheres: for example, products of spheres occur. We will not analyze the topology here, but point out the following elementary 
observations: in [2], the authors introduce the notion of $\Delta_{1}, \ldots, \Delta_{r}$ being $k$-independent ([2], Def. 3.1) Then it follows from the proof of [2], Theorem 3.3 that if $\Delta_{1}, \ldots, \Delta_{r}$ are $k$-independent, $k \geq 3$, then

$$
h^{1}\left(\mathcal{O}_{\mathcal{X}_{0}}\right)=\cdots=h^{k-2}\left(\mathcal{O}_{\mathcal{X}_{0}}\right)=0 .
$$

But by [4], Proposition 2.35, $H^{i}\left(\mathcal{X}_{0}, \mathcal{O}_{\mathcal{X}_{0}}\right)=H^{i}\left(B_{\nabla}, \mathbb{C}\right)$, so if $\Delta_{1}, \ldots, \Delta_{r}$ are $\operatorname{dim} M_{\mathbb{R}}-r+1$ independent, then $B_{\nabla}$ is a rational homology sphere. After this paper was completed, Haase and Zharkov [7] showed that $B_{\nabla}$ is in fact a sphere in this last case.

\section{The Batyrev-Borisov Construction: the general CASE.}

We now proceed to the general case. We would like to allow a more general polarization and degeneration. We are initially given data as in $\S 2: \Delta, \Delta_{i}, \nabla, \nabla_{i}, \Delta^{*}, \nabla^{*}, \varphi=\sum_{i=1}^{r} \varphi_{i}$ and $\check{\varphi}=\sum_{i=1}^{r} \check{\varphi}_{i}$, where $\varphi, \varphi_{i}$ are piecewise linear functions on the normal fan $\Sigma$ to $\Delta$, and $\check{\varphi}, \check{\varphi}_{i}$ are piecewise linear functions on the normal fan $\check{\Sigma}$ to $\nabla$.

We will now consider some additional data: subdivisions $\Sigma^{\prime}$ and $\check{\Sigma}^{\prime}$ of $\Sigma$ and $\check{\Sigma}$ respectively, and functions

$$
\begin{array}{cc}
h: & N_{\mathbb{R}} \rightarrow \mathbb{R} \\
\check{h}: & M_{\mathbb{R}} \rightarrow \mathbb{R}
\end{array}
$$

which are integral piecewise linear and strictly convex on the fans $\Sigma^{\prime}$ and $\check{\Sigma}^{\prime}$ respectively. These define ample divisors on partial resolutions of $\mathbb{P}_{\Delta}$ and $\mathbb{P}_{\nabla}$ respectively. We will make an additional assumption that the functions

$$
\begin{array}{ll}
h^{\prime}:=h-\varphi: & N_{\mathbb{R}} \rightarrow \mathbb{R} \\
\check{h}^{\prime}:=\check{h}-\check{\varphi}: & M_{\mathbb{R}} \rightarrow \mathbb{R}
\end{array}
$$

are convex on $\Sigma^{\prime}$ and $\check{\Sigma}^{\prime}$ respectively (though not necessarily strictly convex).

In general, if $g: N_{\mathbb{R}} \rightarrow \mathbb{R}$ is a piecewise linear convex function on $\Sigma^{\prime}$, write $\Delta^{g}$ for the Newton polytope of $g$, i.e.

$$
\Delta^{g}=\left\{m \in M_{\mathbb{R}} \mid\langle m, n\rangle \geq-g(n) \quad \forall n \in N_{\mathbb{R}}\right\},
$$

and similarly if $\check{g}: M_{\mathbb{R}} \rightarrow \mathbb{R}$ is a piecewise linear convex function on $\check{\Sigma}^{\prime}$, write

$$
\nabla^{\check{g}}=\left\{n \in N_{\mathbb{R}} \mid\langle m, n\rangle \geq-\check{g}(m) \quad \forall m \in M_{\mathbb{R}}\right\} .
$$

For any subset $\tau \subseteq \Delta$ for $\Delta \subseteq M_{\mathbb{R}}$ a polytope, we write

$$
N_{\Delta}(\tau):=\left\{n \in N_{\mathbb{R}}|n|_{\tau} \text { is constant and }\langle m, n\rangle \geq\left\langle m^{\prime}, n\right\rangle \text { for } m \in \Delta, m^{\prime} \in \tau\right\},
$$

the normal cone of $\Delta$ along the subset $\tau$.

Let $\Sigma_{h^{\prime}}^{\prime}$ be the fan of not necessarily strictly convex cones on which $h^{\prime}$ is strictly convex. For example, if $h^{\prime}$ is itself linear, $\Sigma_{h^{\prime}}^{\prime}$ consists only of the cone $N_{\mathbb{R}}$. Similarly define $\check{\Sigma}_{\check{h}^{\prime}}^{\prime}$. 
Cones of $\check{\Sigma}^{\prime}$ are in one-to-one correspondence with faces of $\nabla^{\check{h}}$, and cones of $\check{\Sigma}_{\check{h}^{\prime}}^{\prime}$ are in one-to-one correspondence with faces of $\nabla^{\check{h}^{\prime}}$. Write this correspondence as

$$
\check{\delta}: \check{\Sigma}^{\prime} \rightarrow\left\{\text { faces of } \nabla^{\check{h}}\right\}
$$

and

$$
\check{\delta}_{\check{h}^{\prime}}: \check{\Sigma}_{\check{h}^{\prime}}^{\prime} \rightarrow\left\{\text { faces of } \nabla^{\check{h}^{\prime}}\right\}
$$

Generalising the notions of $\S 2$, if $\sigma \subseteq \partial \Delta^{*}$ is any set, write

$$
\begin{aligned}
\beta_{i}^{*}(\sigma) & =\left\{n \in \sigma \mid \varphi_{i}(n)=1\right\} \subseteq \nabla_{i} \\
\partial(\sigma) & =\beta_{1}^{*}(\sigma)+\cdots+\beta_{r}^{*}(\sigma) \subseteq \nabla
\end{aligned}
$$

and for $\sigma \subseteq \partial \nabla^{*}$

$$
\begin{aligned}
\delta_{i}^{*}(\sigma) & =\left\{m \in \sigma \mid \check{\varphi}_{i}(m)=1\right\} \subseteq \Delta_{i} \\
\beta(\sigma) & =\delta_{1}^{*}(\sigma)+\cdots+\delta_{r}^{*}(\sigma) \subseteq \Delta .
\end{aligned}
$$

The faces of a Minkowski sum $\Delta+\Delta^{\prime}$ are of the form $\sigma+\sigma^{\prime}$ where $\sigma$ and $\sigma^{\prime}$ are faces of $\Delta$ and $\Delta^{\prime}$ respectively, and this decomposition is unique. Then let $\mathscr{R}_{\nabla^{*}}^{h}:=\left\{(\sigma, \tau) \mid \sigma\right.$ is a face of $\nabla^{*}$ with $\beta(\sigma) \neq \emptyset, \tau$ a face of $\Delta^{h^{\prime}}, \sigma+\tau$ a face of $\left.\nabla^{*}+\Delta^{h^{\prime}}\right\}$, $\mathscr{R}_{\Delta^{*}}^{\check{h}}:=\left\{(\sigma, \tau) \mid \sigma\right.$ is a face of $\Delta^{*}$ with $g(\sigma) \neq \emptyset, \tau$ a face of $\nabla^{\check{h}^{\prime}}, \sigma+\tau$ a face of $\left.\Delta^{*}+\nabla^{\check{h}^{\prime}}\right\}$. We then define the underlying topological manifolds which will later acquire affine structures by

$$
\begin{aligned}
& B_{\nabla}^{\check{h}}=\bigcup_{(\sigma, \tau) \in \mathscr{R}_{\Delta}^{\check{h}}} \delta(\sigma)+\tau, \\
& B_{\Delta}^{h}=\bigcup_{(\sigma, \tau) \in \mathscr{R}_{\nabla^{*}}^{h}} \beta(\sigma)+\tau .
\end{aligned}
$$

Lemma 3.1. There is a one-to-one correspondence between proper faces of $\Delta^{*}+\nabla^{\check{h}^{\prime}}$ and elements of the set

$$
\left\{\sigma \cap \tau \mid \sigma \text { is a face of } \Delta, \tau \in \check{\Sigma}_{\check{h}^{\prime}}^{\prime}, \sigma \cap \tau \neq \emptyset\right\} \text {. }
$$

Furthermore, if $\rho$ is an element of this set and $\rho=\sigma \cap \tau$ for $\sigma$ the minimal face of $\Delta$ containing $\rho$ and $\tau$ the minimal cone of $\check{\Sigma}_{\check{h}^{\prime}}^{\prime}$ containing $\rho$, then the corresponding face of $\Delta^{*}+\nabla^{\check{h}^{\prime}}$ is $\sigma^{*}+\check{\delta}_{\check{h}^{\prime}}(\tau)$.

Proof. Let $\psi: M_{\mathbb{R}} \rightarrow \mathbb{R}$ be defined by

$$
\psi(m)=-\inf \left\{\langle m, n\rangle \mid n \in \Delta^{*}\right\},
$$

so that $\Delta^{*}=\left\{n \in N_{\mathbb{R}} \mid\langle m, n\rangle \geq-\psi(m)\right.$ for all $\left.m \in M_{\mathbb{R}}\right\}$. Then by [13], Theorem A.18,

$$
\Delta^{*}+\nabla^{\breve{h}^{\prime}}=\left\{n \in N_{\mathbb{R}} \mid\langle m, n\rangle \geq-\psi(m)-\check{h}^{\prime}(m) \text { for all } m \in M_{\mathbb{R}}\right\} .
$$


Furthermore, it is clear that the coarsest decomposition of $M_{\mathbb{R}}$ into cones on which $\psi+\check{h}^{\prime}$ is linear is $\left\{C(\sigma) \cap \tau \mid \sigma\right.$ is a face of $\left.\Delta, \tau \in \check{\Sigma}_{\check{h}^{\prime}}^{\prime}\right\}$, and by [13], Corollary A.19, there is a oneto-one correspondence between faces of $\Delta^{*}+\nabla^{\check{h}^{\prime}}$ with this set of cones. If we only consider proper faces, then we omit the cone $\{0\}$, and thus the set of proper faces is in one-to-one correspondence with the given set. Explicitly, the face of $\Delta^{*}+\nabla^{\check{h}^{\prime}}$ corresponding to $\sigma \cap \tau$ is

$$
\left\{n \in N_{\mathbb{R}} \mid\langle m, n\rangle \geq-\psi(m)-\check{h}^{\prime}(m) \text { for all } m \in M_{\mathbb{R}} \text { with equality when } m \in C(\sigma) \cap \tau\right\} .
$$

The last statement then follows easily from this.

Lemma 3.2. If $\sigma^{*}+\check{\delta}_{\check{h}^{\prime}}(\tau)$ is a face of $\Delta^{*}+\nabla^{\check{h}^{\prime}}$ with $\rho \neq \emptyset$, then $\rho+\check{\delta}_{\check{h}^{\prime}}(\tau)$ is a face of $\nabla+\nabla^{\check{h}^{\prime}}=\nabla^{\check{h}}$.

Proof. The faces of $\nabla^{\check{h}}$ are in one-to-one correspondence with cones $\rho$ of $\check{\Sigma}^{\prime}$. If $\rho \in \check{\Sigma}^{\prime}$ is given by $\rho=C\left(\varrho^{*}\right) \cap \tau$ for $\varrho^{*}$ a face of $\nabla^{*}$ and $\tau \in \check{\Sigma}_{\check{h}^{\prime}}^{\prime}$, and if $C\left(\varrho^{*}\right)$ and $\tau$ are minimal cones of $\check{\Sigma}$ and $\check{\Sigma}_{\check{h}^{\prime}}^{\prime}$ over $C\left(\rho^{*}\right) \cap \tau$, then the corresponding face of $\nabla^{\check{h}}$ is $\rho+\check{\delta}_{\breve{h}^{\prime}}(\tau)$. Now assume $\sigma^{*}+\check{\delta}_{\check{h}^{\prime}}(\tau)$ is a face of $\Delta^{*}+\nabla^{\check{h}^{\prime}}$. Then $C(\sigma), \tau$ are minimal over $C(\sigma) \cap \tau$. Since $C(\sigma) \cap \tau \subseteq C\left(\rho^{*}\right) \cap \tau, \tau$ must be minimal over $C\left(\rho^{*}\right) \cap \tau$. If there is a smaller face $\mathrm{m}^{*} \subseteq \varrho^{*}$ of $\nabla^{*}$ with $C\left(\rho^{*}\right) \cap \tau=C\left(\mathrm{~m}^{*}\right) \cap \tau$, we wish to show $\sigma \cap \tau=\omega \cap \tau$, therefore violating the assumption that $\sigma$ was minimal over $\sigma \cap \tau$. This follows from

Claim. $\sigma \cap \tau=\left\{m \in C\left(\varrho^{*}\right) \cap \tau \mid \check{\varphi}_{i}(m)=1, \quad 1 \leq i \leq r\right\}$.

To prove the claim, note the left hand side is clearly contained in the right hand side. Conversely, if $m \in C\left(\varrho^{*}\right) \cap \tau$, write $m=\sum_{i} r_{i} m_{i}$ with $m_{i}$ vertices of $\varrho^{*}$ and $r_{i} \geq 0$. Since each $m_{i}$ is an integral point of $\nabla^{*}, \check{\varphi}_{j}\left(m_{i}\right)=0$ or 1 , with 1 occurring for precisely one value of $j$, so by reindexing we can write $m=\sum r_{i j} m_{i j}$ with $\check{\varphi}_{k}\left(m_{i j}\right)=\delta_{i k}$. Thus if $\check{\varphi}_{i}(m)=1$, $\sum_{j} r_{i j}=1$, and $m=\sum_{i=1}^{r} m_{i}^{\prime}$ with $m_{i}^{\prime}=\sum_{j} r_{i j} m_{i j}$ points of $\varrho^{*}$ with $\check{\varphi}_{i}\left(m_{i}^{\prime}\right)=1$. Thus $m \in \sigma$.

Corollary 3.3. $B_{\nabla}^{\check{h}} \subseteq \partial \nabla^{\check{h}}$ and $B_{\Delta}^{h} \subseteq \partial \Delta^{h}$.

Unlike in $\S 2$, we won’t separate the combinatorial description of the affine structures on these two manifolds from the geometric arguments, as these structures depend on some additional choices motivated by the geometry.

In general, $\Delta$ and $\nabla$ define projective toric varieties $\left(\mathbb{P}_{\Delta}, \mathcal{O}_{\mathbb{P}_{\Delta}}(1)\right),\left(\mathbb{P}_{\nabla}, \mathcal{O}_{\mathbb{P}_{\nabla}}(1)\right)$ and $\Delta^{h}$, $\nabla^{\check{h}}$ define (partial crepant resolutions) $\pi: \mathbb{P}_{\Delta^{h}} \rightarrow \mathbb{P}_{\Delta}$ and $\check{\pi}: \mathbb{P}_{\nabla^{\check{h}}} \rightarrow \mathbb{P}_{\nabla}$, where $\mathbb{P}_{\Delta^{h}}, \mathbb{P}_{\nabla^{\check{h}}}$ are toric varieties defined by the fans $\Sigma^{\prime}$ and $\check{\Sigma}^{\prime}$ respectively.

We could then, for example, define a family $\mathcal{X} \rightarrow \mathcal{S}$ with $\mathcal{X} \subseteq \mathbb{P}_{\Delta} \times \mathcal{S}$ with equations

$$
\sum_{m \in \Delta_{1} \cap M} t^{\check{h}(m)} z^{m}=\cdots=\sum_{m \in \Delta_{r} \cap M} t^{\check{h}(m)} z^{m}=0
$$


where $m \in \Delta_{i} \cap M$ gives a monomial $z^{m}$ on the large torus orbit, extending to a section of the line bundle $\mathcal{L}_{i}$ represented by the function $\varphi_{i}$.

There are two problems with this. First, we need to resolve $\mathbb{P}_{\Delta}$ so that we can suitably polarize the family using $h$. Second, the singularities of $\mathcal{X}$ along the fibre with $t=0$ are exceptionally bad, and these must be resolved.

To resolve these issues, we proceed as follows. Let $\tilde{\Delta}_{i} \subseteq \tilde{M}_{\mathbb{R}}=M_{\mathbb{R}} \oplus \mathbb{R}$ be defined as

$$
\tilde{\Delta}_{i}=\left\{(m, l) \in M_{\mathbb{R}} \oplus \mathbb{R} \mid m \in \Delta_{i}, l \geq \check{h}^{\prime}(m)\right\} .
$$

In other words, $\tilde{\Delta}_{i}$ is a polytope extending infinitely upwards but projecting to $\Delta_{i}$. $\tilde{\Delta}_{i}$ has two types of faces: the vertical faces which project to a polytope contained in $\partial \tilde{\Delta}_{i}$ of one smaller dimension, and the lower faces, which project homeomorphically to subpolytopes of $\Delta_{i}$. (We remark that a similar method was used in [9] to obtain examples of semistable degenerations of Calabi-Yau manifolds. To apply that method here, one would use $\check{h}$ instead of $\check{h}^{\prime}$ in the definition of $\tilde{\Delta}_{i}$. We do not use this as this does not yield a toric degeneration.)

Next, let $\tilde{\Delta}=\tilde{\Delta}_{1}+\cdots+\tilde{\Delta}_{r}$. The polytope $\tilde{\Delta}$ again extends infinitely upwards, and projects to $\Delta$. The faces of $\tilde{\Delta}$ are again separated into vertical and lower faces. Let $\tilde{\Sigma}$ be the normal fan to $\tilde{\Delta}$, living in $\tilde{N}_{\mathbb{R}}=N_{\mathbb{R}} \oplus \mathbb{R}$. In other words, for any face $\tilde{\sigma}$ of $\tilde{\Delta}$, we have a cone in $\tilde{\Sigma}$ of the form $N_{\tilde{\Delta}}(\tilde{\sigma})$.

Denote by $X(\tilde{\Sigma})$ the $n+1$-dimensional toric variety defined by the fan $\tilde{\Sigma}$.

Let $t$ denote the monomial function on $X(\tilde{\Sigma})$ given by $(0,1) \in \tilde{M}=M \oplus \mathbb{Z}$. A priori this is a rational function. We have

Proposition 3.4. The function $t$ is regular, inducing a map $f: X(\tilde{\Sigma}) \rightarrow \mathbb{A}^{1}$. Each fibre of $f$ except over $t=0$ is isomorphic to $\mathbb{P}_{\Delta}$.

Proof. There are two sorts of one-dimensional cones in $\tilde{\Sigma}$. One sort are normal to vertical faces of $\tilde{\Delta}$, and these are of the form $(n, 0)$ for $n \in \Sigma(1)$. The other sort are normal to maximal lower faces. Since $(0, l) \in \tilde{\Delta}$ for $l>0$, if $(m, r)$ is a primitive generator of $N_{\tilde{\Delta}}(\tilde{\sigma})$ for a lower face $\tilde{\sigma}$, we must have $r l>0$, so $r>0$. The function $t$ vanishes to order $r$ along the divisor of $X(\tilde{\Sigma})$ corresponding to $(m, r)$; thus in particular $t$ does not have a pole along any divisor of $X(\tilde{\Sigma})$. From this we conclude $t$ is a regular function.

It is easy to see that if we intersect the fan $\tilde{\Sigma}$ with $N_{\mathbb{R}}$ we obtain the fan $\Sigma$. The second statement then follows from the exact sequence

$$
0 \rightarrow N_{\mathbb{R}} \rightarrow \tilde{N}_{\mathbb{R}} \rightarrow \mathbb{R} \rightarrow 0
$$

and corresponding maps of fans of $\Sigma$ in $N_{\mathbb{R}}$ to $\tilde{\Sigma}$ in $\tilde{N}_{\mathbb{R}}$, and $\tilde{\Sigma}$ to the fan in $\mathbb{R}$ defining $\mathbb{A}^{1}$. The second map of fans induces the map $f$, and the first map embeds $\mathbb{P}_{\Delta}$ in $X(\tilde{\Sigma})$ as a general fibre of the map $f$. 
We now need a more detailed description of the fan $\tilde{\Sigma}$, and hence a more detailed description of $\tilde{\Delta}$. We do this as follows.

\section{Proposition 3.5.}

$$
\tilde{\Delta}=\left\{(m, l) \mid m \in \Delta, l \geq \check{h}^{\prime}(m)\right\} .
$$

In particular, there is a one-to-one correspondence between maximal lower faces of $\tilde{\Delta}$ and maximal cones $\sigma \in \check{\Sigma}_{\check{h}^{\prime}}$, with

$$
\left\{\left(m, \check{h}^{\prime}(m)\right) \mid m \in \sigma \cap \Delta\right\}
$$

the maximal lower face of $\tilde{\Delta}$ corresponding to $\sigma \in \check{\Sigma}_{\check{h}^{\prime}}$.

Proof. It is clear that

$$
\tilde{\Delta} \subseteq\left\{(m, l) \mid m \in \Delta, l \geq \check{h}^{\prime}(m)\right\} ;
$$

indeed, if $(m, l) \in \tilde{\Delta}$, then $(m, l)=\sum_{i=1}^{r}\left(m_{i}, l_{i}\right)$ for some $\left(m_{i}, l_{i}\right) \in \tilde{\Delta}_{i}$, so $l_{i} \geq \check{h}^{\prime}\left(m_{i}\right)$. But by convexity of $\check{h}^{\prime}$,

$$
\check{h}^{\prime}(m) \leq \sum \check{h}^{\prime}\left(m_{i}\right) \leq \sum_{i=1}^{r} l_{i}=l .
$$

We only need to show the converse inclusion now. To do this, let $\sigma$ be any maximal cone of $\check{\Sigma}^{\prime}$, set

$$
\begin{aligned}
\tau_{i} & :=\delta_{i}^{*}\left(\sigma \cap \partial \nabla^{*}\right) \\
\sigma_{i} & :=\operatorname{Conv}\left\{0, \tau_{i}\right\}
\end{aligned}
$$

and set $\sigma^{\prime}:=\sigma_{1}+\cdots+\sigma_{r}$.

Claim 3.6. $\sigma^{\prime}=\sigma \cap \Delta$.

Proof. Since $\Delta=\Delta_{1}+\cdots+\Delta_{r}$, it is clear that $\sigma^{\prime} \subseteq \sigma \cap \Delta$. If $m \in \sigma_{i}$ then $\operatorname{lm} \in \sigma_{i}$ for $0 \leq l \leq 1$. Thus $\operatorname{Conv}\left\{\sigma_{1}, \ldots, \sigma_{r}\right\} \subseteq \sigma_{1}+\cdots+\sigma_{r}$. On the other hand, $\sigma \cap \partial \nabla^{*}$ is the convex hull of some integral points; let $v$ be such an integral point. Then $\check{\varphi}_{i}(v)=1$ for some $i$, so $v \in \sigma_{i}$ for some $i$. Thus $\operatorname{Conv}\left\{\sigma_{1}, \ldots, \sigma_{r}\right\}=\sigma \cap \nabla^{*}$. So we have

$$
\sigma \cap \nabla^{*} \subseteq \sigma^{\prime} \subseteq \sigma \cap \Delta
$$

Now let $\rho$ be a maximal face of $\sigma^{\prime}$ not contained in a face of $\sigma$. If we can prove $\rho \subseteq \partial \Delta$, then we will obtain the equality $\sigma^{\prime}=\sigma \cap \Delta$. It is clear that $0 \notin \rho$ since $\sigma \cap \nabla^{*} \subseteq \sigma^{\prime}$. Since $\rho=\rho_{1}+\cdots+\rho_{r}$ where $\rho_{i}$ is a face of $\sigma_{i}$, we must have $0 \notin \rho_{i}$ for at least one $i$.

Since $\sigma$ is maximal, there exists a unique vertex $n$ of $\nabla$ such that $\langle n, m\rangle=-1$ for all $m \in \sigma \cap \partial \nabla^{*}$, and since $\nabla=\nabla_{1}+\cdots+\nabla_{r}, n$ decomposes uniquely as $n=n_{1}+\cdots+n_{r}$ with $n_{i}$ a vertex of $\nabla_{i} \subseteq \Delta^{*}$ and $n_{i}$ defines the function $-\check{\varphi}_{i}$ on $\sigma$. Now $\tau_{i}$ is the unique 
maximal face of $\sigma_{i}$ not containing 0 (note for some $i, \tau_{i}$ may be empty, but this is only the case when $\left.\sigma_{i}=\{0\}\right)$. Thus $\rho_{i} \subseteq \tau_{i}$ so

$$
\rho \subseteq \sigma_{1}+\cdots+\tau_{i}+\cdots+\sigma_{r}
$$

However $n_{i} \in \Delta^{*}$, and $\left\langle n_{i}, \sigma_{j}\right\rangle=0$, so $\left\langle n_{i}, m\right\rangle=-1$ for all $m \in \rho$. Thus $\rho$ is contained in $\partial \Delta$.

Now for any $m \in \Delta$, there is some maximal $\sigma \in \check{\Sigma}^{\prime}$ such that $m \in \sigma$, and thus $m \in \sigma^{\prime}$ by the claim. Then $m=\sum m_{i}$ with $m_{i} \in \sigma_{i}$ and $\left(m, \check{h}^{\prime}(m)\right)=\sum\left(m_{i}, \check{h}^{\prime}\left(m_{i}\right)\right) \in \tilde{\Delta}$ as $m_{1}, \ldots, m_{r} \in \sigma$ on which $\check{h}^{\prime}$ is linear.

\section{Proposition 3.7.}

$$
\begin{aligned}
\tilde{\Sigma} & =\left\{C\left(\sigma^{*}\right) \times\{0\} \mid \sigma^{*} \text { a face of } \Delta^{*}\right\} \\
& \cup\left\{C\left(\sigma^{*}\right) \times\{0\}+C(\tau \times\{1\}) \mid \begin{array}{r}
\sigma^{*} \text { a face of } \Delta^{*}, \tau \text { a face of } \nabla^{\check{h}^{\prime}}, \\
\text { and } \sigma^{*}+\tau \text { a face of } \Delta^{*}+\nabla^{\check{h}^{\prime}}
\end{array}\right\} \\
& \cup\left\{C(\tau \times\{1\}) \mid \tau \text { a face of } \nabla^{\check{h}^{\prime}}\right\} .
\end{aligned}
$$

Here $C$ denotes the cone over a set with vertex the origin, as usual.

Proof. The normal cone to a vertical face of $\tilde{\Delta}$ mapping to a face $\sigma$ of $\Delta$ is just $C\left(\sigma^{*}\right) \times\{0\}$. A lower face $\rho$ of $\tilde{\Delta}$ is projected either to a subpolytope of $\Delta$ containing 0 , or is mapped to a polytope contained in $\partial \Delta$. In the former case, $\rho$ maps to $\tau \cap \Delta$ for some $\tau \in \check{\Sigma}_{\check{h}^{\prime}}^{\prime}$. The maximal faces of $\tilde{\Delta}$ containing $\rho$ are then in one-to-one correspondence with maximal cones $\tau^{\prime}$ of $\check{\Sigma}_{\check{h}^{\prime}}^{\prime}$ containing $\tau$, and then the normal cone to $\tilde{\Delta}$ along $\rho$ is generated by the set of normal vectors to the maximal faces of $\tilde{\Delta}$ containing $\rho$, and this is

$$
\left\{\left(-n_{\tau^{\prime}}^{\prime}, 1\right) \mid \tau^{\prime} \in \Sigma_{\check{h}^{\prime}}^{\prime} \text { maximal cones containing } \tau\right\}
$$

where $n_{\tau^{\prime}}^{\prime}$ defines $\check{h}^{\prime}$ on $\tau^{\prime}$. But $\check{\delta}_{\check{h}^{\prime}}(\tau)$ is the convex hull of

$$
\left\{-n_{\tau^{\prime}}^{\prime} \mid \tau^{\prime} \in \Sigma_{\check{h}^{\prime}}^{\prime} \text { a maximal cone containing } \tau\right\},
$$

so the normal cone to $\tilde{\Delta}$ along $\rho$ is $C\left(\check{\delta}_{\breve{h}^{\prime}}(\tau) \times\{1\}\right)$. In the second case, $\rho$ is the intersection of some minimal vertical face containing it, mapping, say, to a face $\sigma$ of $\Delta$, and a minimal horizontal face containing both $\rho$ and 0 , mapping to $\tau \cap \Delta$ for some $\tau \in \check{\Sigma}_{\check{h}^{\prime}}^{\prime}$. In this case, the normal cone is

$$
C\left(\sigma^{*}\right) \times\{0\}+C\left(\check{\delta}_{\check{h}^{\prime}}(\tau) \times\{1\}\right) .
$$

But the set of such $\rho$ is clearly in one-to-one correspondence with the set defined in Lemma 3.1 , so the result follows.

The polytope $\tilde{\Delta}$ determines a line bundle $\tilde{\mathcal{L}}$ on $X(\tilde{\Sigma})$. This line bundle is induced by the piecewise linear function $\tilde{\varphi}$ on $\tilde{\Sigma}$ defined by

$$
\tilde{\varphi}(\tilde{n})=-\inf \{\langle\tilde{n}, \tilde{m}\rangle \mid \tilde{m} \in \tilde{\Delta}\} .
$$


We clearly have $\tilde{\varphi}(n, 0)=1$ for $n \in \partial \Delta^{*}$, and since $(0,0)$ is in every maximal lower face of $\tilde{\Delta}, \tilde{\varphi}(n, 1)=0$ for every $n \in \nabla^{\check{h}^{\prime}}$. Also, the Minkowski decomposition $\tilde{\Delta}=\tilde{\Delta}_{1}+\cdots+\tilde{\Delta}_{r}$ induces a decomposition $\tilde{\mathcal{L}}=\tilde{\mathcal{L}}_{1} \otimes \cdots \otimes \tilde{\mathcal{L}}_{r}$. This induces a decomposition $\tilde{\varphi}=\tilde{\varphi}_{1}+\cdots+\tilde{\varphi}_{r}$, with $\tilde{\varphi}_{i}(n, 0)=\varphi_{i}(n)$ and $\tilde{\varphi}_{i}(n, 1)=0$ for any $n \in \nabla^{\check{h}^{\prime}}$.

Definition 3.8. A subdivision $\tilde{\Sigma}^{\prime}$ of the fan $\tilde{\Sigma}$ is $\operatorname{good}$ for $\Sigma^{\prime}$ if the fan $\left\{\sigma \cap\left(N_{\mathbb{R}} \times\{0\}\right) \mid \sigma \in\right.$ $\left.\tilde{\Sigma}^{\prime}\right\}$ coincides with $\Sigma^{\prime}$ and furthermore, every one-dimensional cone of $\tilde{\Sigma}^{\prime}$ not contained in $N_{\mathbb{R}} \times\{0\}$ is generated by a primitive vector $(n, 1)$ with $n \in \nabla^{\check{h}^{\prime}}$.

Observation 3.9. If $\tilde{\Sigma}^{\prime}$ is a good subdivision for $\Sigma^{\prime}$, it contains three sorts of cones:

(1) Cones of the form $C(\sigma) \times\{0\}$ for $C(\sigma) \in \Sigma^{\prime}$ with $\sigma \subseteq \partial \Delta^{*}$;

(2) Cones of the form $C(\sigma) \times\{0\}+C(\tau \times\{1\})$ where $C(\sigma) \in \Sigma^{\prime}$ for $\sigma \subseteq \partial \Delta^{*}, \tau \subseteq \partial \nabla^{\check{h}^{\prime}}$, $\sigma+\tau$ contained in a face of $\Delta^{*}+\nabla^{\breve{h}^{\prime}}$.

(3) Cones contained in $C\left(\nabla^{\check{h}^{\prime}} \times\{1\}\right)$.

We will call a cone of $\tilde{\Sigma}^{\prime}$ relevant if it is of the second type with $\delta(\sigma) \neq \emptyset$.

Given a subdivision, we obtain a (partial) resolution $\tilde{\pi}: X\left(\tilde{\Sigma}^{\prime}\right) \rightarrow X(\tilde{\Sigma})$. There is a function $f^{\prime}: X\left(\tilde{\Sigma}^{\prime}\right) \rightarrow \mathbb{A}^{1}\left(f^{\prime}=f \circ \tilde{\pi}\right)$, again coming from the monomial $t$, whose general fibre, as in Proposition 3.4, is isomorphic to $\mathbb{P}_{\Delta^{h}}$.

Let $\tilde{\mathcal{L}}_{i}^{\prime}=\tilde{\pi}^{*} \tilde{\mathcal{L}}_{i}$. The functions $\tilde{\varphi}_{i}$ are the piecewise linear functions on $\tilde{\Sigma}^{\prime}$ determining $\tilde{\mathcal{L}}_{i}^{\prime}$ also, and again the Newton polytopes of $\tilde{\mathcal{L}}_{i}^{\prime}$ are $\tilde{\Delta}_{i}$.

Let $s_{i} \in \Gamma\left(X\left(\tilde{\Sigma}^{\prime}\right), \tilde{\mathcal{L}}_{i}^{\prime}\right)$ be a general section, and let $s_{i}^{0} \in \Gamma\left(X\left(\tilde{\Sigma}^{\prime}\right), \tilde{\mathcal{L}}_{i}^{\prime}\right)$ be the section defined by $(0,0) \in \tilde{\Delta}_{i}$. Thus in particular $t s_{i}$ is also a section of $\tilde{\mathcal{L}}_{i}$. Let $\mathcal{X}^{\prime} \subseteq X\left(\tilde{\Sigma}^{\prime}\right)$ be defined by the equations

$$
t s_{1}+s_{1}^{0}=\cdots=t s_{r}+s_{r}^{0}=0
$$

in $X\left(\tilde{\Sigma}^{\prime}\right)$. We obtain by restriction $f^{\prime}: \mathcal{X}^{\prime} \rightarrow \mathbb{A}^{1}$. We can replace $\mathcal{X}^{\prime}$ with a family over $\mathcal{S}=\operatorname{Spec} R$, where $R$ is a discrete valuation ring with uniformizing parameter $t$, via basechange.

Theorem 3.10. $f^{\prime}: \mathcal{X}^{\prime} \rightarrow \mathcal{S}$ is a toric degeneration of Calabi-Yau manifolds.

Proof. We follow the same outline as the proof of Proposition 2.16. First let us check that $\mathcal{X}^{\prime}$ is irreducible, $f^{\prime}: \mathcal{X}^{\prime} \rightarrow \mathcal{S}$ flat of relative dimension $n-r$. If $\eta$ is the generic point of $\mathcal{S}$, then $\mathcal{X}_{\eta}^{\prime} \subseteq \mathbb{P}_{\Delta^{h}} \times \eta$ is cut out by generic sections of $\pi^{*} \mathcal{L}_{1}, \ldots, \pi^{*} \mathcal{L}_{r}$, and hence is a codimension $n-r$ complete intersection with canonical singularities. We need to check that $\operatorname{dim} \mathcal{X}_{0}^{\prime}=n-r$. Now $\mathcal{X}_{0}^{\prime}$ is defined by the equations

$$
t=s_{1}^{0}=\cdots=s_{r}^{0}=0
$$

in $X\left(\tilde{\Sigma}^{\prime}\right)$. Each of these equations defines a toric divisor, so each irreducible component of $\mathcal{X}_{0}^{\prime}$ is a toric stratum. Let $\rho$ be a cone in $\tilde{\Sigma}^{\prime}$ corresponding to a toric stratum of $\mathcal{X}_{0}^{\prime}$. Then 
none of $\tilde{\varphi}_{i}$ or $(0,1)$ can be identically zero on the cone $\rho$. Thus following Observation 3.9, $\rho$ cannot be of the first or third type, since $(0,1)$ vanishes on cones of the first type and $\tilde{\varphi}_{i}$ vanishes on cones of the third type. Thus we can write

$$
\rho=C(\sigma) \times\{0\}+C(\tau \times\{1\})
$$

with $\sigma \subseteq \partial \Delta^{*}$. In addition, we must have $\delta(\sigma) \neq \emptyset$, since for each $i, \tilde{\varphi}_{i}$ must take the value 1 on some vertex of $\sigma$. Thus $\rho$ is a relevant cone.

Now suppose $\rho$ corresponds to an irreducible component of $\mathcal{X}_{0}^{\prime}$. Then $\sigma$ has vertices $n_{1}, \ldots, n_{r}$ with $\varphi_{i}\left(n_{j}\right)=\delta_{i j}$. Thus as in the proof of Proposition 2.16, $n_{1}, \ldots, n_{r}$ are linearly independent, so $\operatorname{dim} \rho \geq r+1$. Thus the dimension of the corresponding irreducible component is $\leq n-r$. Again by the unmixedness theorem, it must be dimension $n-r$. (Any toric variety is Cohen-Macaulay.) Thus we can conclude as in Proposition 2.16 that $\mathcal{X}^{\prime}$ is irreducible. Thus $f^{\prime}: \mathcal{X}^{\prime} \rightarrow \mathcal{S}$ is proper and flat, and $\mathcal{X}^{\prime}$ is irreducible, and (1) of Definition 1.1 holds.

Next we define the set $Z_{0}$ as

$$
Z_{0}=\operatorname{Sing}\left(\mathcal{X}_{0}^{\prime}\right) \cap \bigcup_{i=1}^{r}\left\{s_{i}=0\right\} .
$$

Since the $s_{i}$ are general, they do not vanish identically on any toric stratum.

We will now consider (4) of Definition 1.1. Let $\bar{x} \rightarrow \mathcal{X}_{0}^{\prime}$ be a geometric point in $\mathcal{X}_{0}^{\prime}$ where none of the $s_{i}$ 's vanish, with image $x \in \mathcal{X}_{0}^{\prime}$. Then the closure of the torus orbit of $x$ in $X\left(\tilde{\Sigma}^{\prime}\right)$ is a toric stratum corresponding to some cone $\rho$ of $\tilde{\Sigma}^{\prime}$. This cone $\rho$ induces an affine open subset

$$
U_{\rho}=\operatorname{Spec} k\left[\rho^{\vee} \cap(M \oplus \mathbb{Z})\right]
$$

of $X\left(\tilde{\Sigma}^{\prime}\right)$. On $U_{\rho}$, we can view $s_{i}$ and $s_{i}^{0}$ as regular functions. Explicitly, if $\left(m_{i}, l_{i}\right) \in M_{\mathbb{R}} \oplus \mathbb{R}$ are chosen to define the function $\tilde{\varphi}_{i}$ on the cone $\rho$ (with $m_{i}$ defining $\varphi_{i}$ on $\rho \cap N_{\mathbb{R}}$ ) then thinking of $z^{\left(m_{i}, l_{i}\right)}$ as a monomial function on $U_{\rho}$, we can write the equation of $\mathcal{X}^{\prime}$ in $U_{\rho}$ as

$$
t f_{i}-z^{\left(m_{i}, l_{i}\right)}=0, \quad i=1, \ldots, r
$$

with $f_{i}$ proportional to $s_{i}$. Since all the $f_{i}$ 's are non-zero at $x$, a neighbourhood of $x$ in $\mathcal{X}^{\prime}$ is locally étale equivalent (as in the proof of Proposition 2.16) to the subscheme of $U_{\rho}$ defined by

$$
t-z^{\left(m_{i}, l_{i}\right)}=0, \quad i=1, \ldots, r .
$$

This is a toric subvariety $U_{\rho^{\prime}}$ of $U_{\rho}$, defined by the following cone. Let $V_{\rho} \subseteq M_{\mathbb{R}} \oplus \mathbb{R}$ be the subspace spanned by $\left\{\left(m_{i}, l_{i}-1\right) \mid 1 \leq i \leq r\right\}$. Then the cone $\rho^{\prime}:=\rho \cap V_{\rho}^{\perp}$ defines an $n-r+1$ dimensional toric variety $U_{\rho^{\prime}}$. In addition, the map $f^{\prime}$ is étale equivalent to the induced map $U_{\sigma^{\prime}} \rightarrow$ Spec $k[t]$ defined by $t$.

Claim 3.11. $\rho^{\prime}=C((g(\sigma)+\tau) \times\{1\})$. 
Proof. Clearly

$$
\rho^{\prime}=\left\{(n, l) \in \rho \mid \tilde{\varphi}_{i}(n, l)=l \quad \forall i\right\} .
$$

Write $(n, l) \in \rho$ as $(n, l)=\left(n^{\prime}, 0\right)+l\left(n^{\prime \prime}, 1\right)$ with $n^{\prime} \in C(\sigma)$ and $n^{\prime \prime} \in \tau$. Then $(n, l) \in \rho^{\prime}$ if and only if $\varphi_{i}\left(n^{\prime}\right)=l$ for all $i$. Now suppose $n^{\prime}$ is a vertex of $g(\sigma)$. Then $n^{\prime}=n_{1}+\cdots+n_{r}$, with $n_{i} \in \beta_{i}^{*}(\sigma)$, so $\varphi_{i}\left(n_{j}\right)=\delta_{i j}$. Thus $\varphi_{i}\left(n^{\prime}\right)=1$, so $\left(n^{\prime}, 0\right)+\left(n^{\prime \prime}, 1\right) \in \rho^{\prime}$ for any $n^{\prime \prime} \in \tau$. Thus $C((\delta(\sigma)+\tau) \times\{1\}) \subseteq \rho^{\prime}$. Conversely, suppose $(n, l) \in \rho^{\prime}$. If $l=0$, then $\varphi_{i}(n)=0$ for all $i$, so $\varphi(n)=0$. But as $\varphi$ takes the constant value 1 on $\partial \Delta^{*}$, this implies $n=0$. Thus we can assume $l \neq 0$, and by dividing by $l$, assume $(n, 1) \in \rho^{\prime}$. Of course, we have $(n, 1)=\left(n^{\prime}, 0\right)+\left(n^{\prime \prime}, 1\right)$ as above, and we just need to show $n^{\prime} \in \delta(\sigma)$. Write $n^{\prime}=\sum r_{j} n_{j}$ for $n_{j}$ vertices of $\sigma, r_{j}>0$. Then as each $n_{j}$ is integral, we have $\varphi_{i}\left(n_{j}\right)=0$ or 1 , and since $\varphi=\sum \varphi_{i}, \varphi_{i}\left(n_{j}\right)=1$ for some $i$. Thus, reindexing, we can write $n^{\prime}=\sum_{(i, j) \in I} r_{i j} n_{i j}$ with $\varphi_{k}\left(n_{i j}\right)=\delta_{i k}$. Thus $\varphi_{i}\left(n^{\prime}\right)=1$ implies $\sum_{j} r_{i j}=1$, so $\sum_{j} r_{i j} n_{i j} \in \beta_{i}^{*}(\sigma)$. Thus $n^{\prime} \in \delta(\sigma)$ as desired.

To conclude the proof of Condition (4) of Definition 1.1, we just have to observe that from the claim, the function $t$ vanishes on every toric divisor of $U_{\rho^{\prime}}$, in fact to order 1 , so in addition $\mathcal{X}_{0}$ is reduced. Then taking $Z=Z_{0} \cup \overline{\operatorname{Sing}\left(\mathcal{X}_{\eta}^{\prime}\right)}, Z$ satisfies Condition (4).

Finally, to check (2) and (3), the key point is to make sure that for any irreducible component $X$ of $\mathcal{X}_{0}^{\prime}$, and toric divisor $D \subseteq X$, there exists another irreducible component $Y$ of $\mathcal{X}_{0}^{\prime}$ with $X \cap Y=D$. However, this is easily verified.

We can now identify the relevant polyhedral decomposition.

Proposition 3.12.

$$
B_{\nabla}^{\breve{h}}=\bigcup \partial(\sigma)+\tau,
$$

where the union is over all relevant cones $C(\sigma) \times\{0\}+C(\tau \times\{1\})$ of a subdivision $\tilde{\Sigma}^{\prime}$ of $\tilde{\Sigma} \operatorname{good}$ for $\Sigma^{\prime}$.

Proof. The inclusion of the right-hand set in the left-hand set is clear. Conversely, let $n \in B_{\nabla}^{\breve{h}}$. Then $n \in \delta(\sigma)+\tau$ for a cone $C(\sigma) \times\{0\}+C(\tau \times\{1\})$ of $\tilde{\Sigma}$, and thus $(n, 1)$ is in a cone $C\left(\sigma^{\prime}\right) \times\{0\}+C\left(\tau^{\prime} \times\{1\}\right)$ of $\tilde{\Sigma}^{\prime}$. We only need to show $n \in \delta\left(\sigma^{\prime}\right)+\tau^{\prime}$. Certainly $(n, 1)=\left(n^{\prime}, 0\right)+\left(n^{\prime \prime}, 1\right)$ for $n^{\prime} \in C\left(\sigma^{\prime}\right), n^{\prime \prime} \in \tau^{\prime}$. Also, it follows from Claim 3.11 applied to $\tilde{\Sigma}$ rather than $\tilde{\Sigma}^{\prime}$, that $\tilde{\varphi}_{i}(n, 1)=1$ for all $i$, so $\varphi_{i}\left(n^{\prime}\right)=1$ for all $i$. But we can then conclude, just as in the proof of the claim, that $n^{\prime} \in \delta\left(\sigma^{\prime}\right)$.

Definition 3.13. We set

$$
\mathscr{P} \tilde{\Sigma}_{\nabla}^{\prime}:=\left\{\delta(\sigma)+\tau \mid C(\sigma) \times\{0\}+C(\tau \times\{1\}) \text { is a relevant cone of } \tilde{\Sigma}^{\prime}\right\} .
$$

This is clearly a polyhedral decomposition of $B_{\nabla}^{\breve{h}}$.

We can then define as usual $\Gamma_{\nabla}^{\tilde{\Sigma}^{\prime}} \subseteq B_{\nabla}^{\check{h}}$ the union of all simplices of $\operatorname{Bar}\left(\mathscr{P} \tilde{\Sigma}_{\nabla}^{\prime}\right) \operatorname{not}$ containing a vertex of $\mathscr{P} \tilde{\Sigma}_{\nabla}^{\prime}$ or intersecting the interior of a maximal face of $\mathscr{P} \tilde{\Sigma}_{\nabla}^{\prime}$. We define an integral affine structure on $B_{\nabla}^{\check{h}} \backslash \Gamma_{\nabla}^{\tilde{\Sigma}^{\prime}}$ as follows. 
For every maximal face $\sigma \in \mathscr{P} \tilde{\Sigma}_{\nabla}^{\prime}$ of $B$, let $\mathbb{A}_{\sigma}$ be the affine subspace of $N_{\mathbb{R}}$ spanned by $\sigma$. Then we define an affine chart on $\operatorname{Int}(\sigma)$ by $\psi_{\sigma}: \operatorname{Int}(\sigma) \hookrightarrow \mathbb{A}_{\sigma}$.

Secondly, consider a vertex $v$ of $\mathscr{P} \nabla \tilde{\Sigma}^{\prime}$. Then $v$ arises from a relevant cone $C(\sigma) \times\{0\}+$ $C(\tau \times\{1\})$ with $\{v\}=g(\sigma)+\tau$. Then clearly $\operatorname{dim} \tau=\operatorname{dim} \delta(\sigma)=0$, so $\tau=\left\{v^{\prime}\right\}$ and $\sigma=\operatorname{Conv}\left\{n_{1}, \ldots, n_{r}\right\}$ where $\varphi_{i}\left(n_{j}\right)=\delta_{i j}$. Clearly $n_{1}, \ldots, n_{r}$ are linearly independent. Letting $W_{v}$ be the union of interiors of all simplices of $\operatorname{Bar}\left(\mathscr{P}_{\nabla}^{\tilde{\Sigma}^{\prime}}\right)$ containing $v$, we define a chart

$$
\psi_{v}: W_{v} \rightarrow N_{\mathbb{R}} / \operatorname{Span}\left(n_{1}, \ldots, n_{r}\right)
$$

via projection.

Proposition 3.14. The charts $\psi_{v}$ and $\psi_{\sigma}$ define an integral affine structure on $B_{\nabla}^{\check{h}} \backslash \Gamma_{\nabla}^{\tilde{\Sigma}^{\prime}}$, making $B_{\nabla}^{\breve{h}}$ an integral affine manifold with singularities of dimension $\operatorname{dim} M_{\mathbb{R}}-r$. In addition, this structure makes $\left(B_{\nabla}^{\check{h}}, \mathscr{P} \tilde{\nabla}^{\prime}\right)$ the dual intersection complex of the degeneration $f^{\prime}: \mathcal{X}^{\prime} \rightarrow \mathcal{S}$.

Proof. Let $v$ be a vertex of $\mathscr{P} \tilde{\Sigma}_{\nabla}^{\prime}$ corresponding to a cone

$$
\omega_{v}=C\left(\operatorname{Conv}\left\{n_{1}, \ldots, n_{r}\right\}\right) \times\{0\}+C(\{w\} \times\{1\})
$$

of $\tilde{\Sigma}^{\prime}$, with $v=n_{1}+\cdots+n_{r}+w$, and let $\rho$ be a maximal facet of $\mathscr{P} \tilde{\Sigma}_{\nabla}^{\prime}$ containing $v$, corresponding to a maximal cone

$$
\omega_{\rho}=C(\sigma) \times\{0\}+C(\tau \times\{1\})
$$

of $\tilde{\Sigma}^{\prime}$ with $\rho=g(\sigma)+\tau$. Then by Claim 3.11,

$$
\mathbb{A}_{\sigma}=\left\{n \in N_{\mathbb{R}} \mid(n, 1) \in V_{\rho}^{\perp}\right\} .
$$

More explicitly, if $\left(m_{i}, l_{i}\right)$ defines $\tilde{\varphi}_{i}$ on $\omega_{\rho}$, then $-\left(m_{i}, l_{i}\right)$ is a vertex of $\tilde{\Delta}_{i}$, and in particular is in a lower face of $\tilde{\Delta}_{i}$, so $l_{i}=-\check{h}^{\prime}\left(m_{i}\right)$. Then

$$
\mathbb{A}_{\sigma}=\left\{n \in N_{\mathbb{R}} \mid\left\langle m_{i}, n\right\rangle=\check{h}\left(m_{i}\right), i=1, \ldots, r\right\} .
$$

Now $\left\langle m_{i}, n_{j}\right\rangle=\delta_{i j}$, so if $\sum a_{j} n_{j} \in \mathbb{A}_{\sigma}$, we have $a_{i}=\left\langle m_{i}, \sum a_{j} n_{j}\right\rangle=\check{h}\left(m_{i}\right)$, so

$$
\mathbb{A}_{\sigma} \cap \operatorname{Span}\left(n_{1}, \ldots, n_{r}\right)=\left\{\sum \check{h}\left(m_{i}\right) m_{i}\right\} .
$$

In particular, the projection $\mathbb{A}_{\sigma} \rightarrow N_{\mathbb{R}} / \operatorname{Span}\left(n_{1}, \ldots, n_{r}\right)$ is an affine isomorphism, with the linear part preserving the integral structure.

We then have to show that $\psi_{v}$ is one-to-one. First let's look at the tangent wedge to $\psi_{v}(\rho)$ at $\psi_{v}(v) \equiv w \bmod \operatorname{Span}\left(n_{1}, \ldots, n_{r}\right)$. As in $\S 2$, it is easy to see that the tangent wedge of $\psi_{v}(\rho)$ at $\psi_{v}(v)$ is the cone $\left(\omega_{\rho}+\mathbb{R} \omega_{v}\right) / \mathbb{R} \omega_{v}$ of the fan $\tilde{\Sigma}^{\prime}\left(\omega_{v}\right)$. Since the maximal facets of $\mathscr{P} \tilde{\Sigma}_{\nabla}^{\prime}$ containing $v$ are in one-to-one correspondence with maximal cones of $\tilde{\Sigma}^{\prime}$ containing $\omega_{v}$, we see that the tangent wedges to all the $\psi_{v}(\rho)$ 's fit together to form the fan $\tilde{\Sigma}^{\prime}\left(\omega_{v}\right)$. In particular $\psi_{v}$ is a $\left(C^{0}\right)$ immersion. 
It is now easy to see $B_{\nabla}^{\check{h}}, \mathscr{P} \underset{\nabla}{\tilde{\Sigma}^{\prime}}$ is the dual intersection complex of the toric degeneration $f^{\prime}: \mathcal{X}^{\prime} \rightarrow S$ as in Proposition 2.16.

We compute monodromy.

Proposition 3.15. Let $v$ and $v^{\prime}$ be two vertices of $\mathscr{P} \tilde{\Sigma}^{\prime}$ and let $\rho$ and $\rho^{\prime}$ be two maximal facets of $\mathscr{P} \tilde{\Sigma}^{\prime}$ containing $v$ and $v^{\prime}$ coming from cones $\omega_{\rho}$ and $\omega_{\rho^{\prime}}$ of $\tilde{\Sigma}^{\prime}$ respectively. Let $\gamma$ be a simple loop based at $v$, passing successively into $\operatorname{Int}(\rho)$, through $v^{\prime}$, into $\operatorname{Int}\left(\rho^{\prime}\right)$ and back to $v$. Write $v=n_{1}+\cdots+n_{r}+w$ and $v^{\prime}=n_{1}^{\prime}+\cdots+n_{r}^{\prime}+w^{\prime}$, and let $\left(m_{1}, l_{1}\right), \ldots,\left(m_{r}, l_{r}\right),\left(m_{1}^{\prime}, l_{1}^{\prime}\right), \ldots,\left(m_{r}^{\prime}, l_{r}^{\prime}\right) \in M$ define the functions $-\tilde{\varphi}_{i}$ on $\omega_{\rho}$ and $\omega_{\rho^{\prime}}$ respectively. Identifying $\Lambda_{v}$ with $N /\left(N \cap \operatorname{Span}\left(n_{1}, \ldots, n_{r}\right)\right)$, then parallel transport $T_{\gamma}: \Lambda_{v} \rightarrow \Lambda_{v}$ around $\gamma$ is given by

$$
T_{\gamma}(n)=n+\sum_{j=1}^{r}\left\langle m_{j}^{\prime}-m_{j}, n\right\rangle\left(n_{j}^{\prime}-n_{j}\right) .
$$

Proof. This proof is identical to that of Proposition 2.13

We now determine when $B$ is simple. (See [4], Definition 1.60). We do this both so we know when Theorem 0.1 can be applied and to justify the relevance of the simplicity condition. Recall that an MPCP (maximal projective crepant partial) resolution of $\mathbb{P}_{\Delta}$ is obtained by choosing $h$ so that the induced decomposition of $\partial \Delta^{*}$ consists only of elementary simplices, i.e. simplices whose only integral points are the vertices.

Theorem 3.16. If $h$ and $\check{h}$ induce $M P C P$ resolutions of $\mathbb{P}_{\Delta}$ and $\mathbb{P}_{\nabla}$ respectively, then $\left(B_{\nabla}^{\check{h}}, \mathscr{P} \underset{\nabla}{\tilde{\Sigma}^{\prime}}\right)$ is simple.

Proof. We follow the notation of [4], §1.5. Let $\tau \in \mathscr{P} \mathscr{\Sigma}_{\nabla}^{\tilde{\Sigma}^{\prime}}, 1 \leq \operatorname{dim} \tau \leq n-r-1$,

$$
\begin{aligned}
\mathscr{P}_{1}(\tau) & =\{e: \omega \rightarrow \tau \mid \operatorname{dim} \omega=1\} \\
\mathscr{P}_{n-r-1}(\tau) & =\{f: \tau \rightarrow \rho \mid \operatorname{dim} \rho=n-r-1\} .
\end{aligned}
$$

(Note that in our case, using morphisms $e: \omega \rightarrow \tau$, etc. is superfluous; whenever $\omega \subseteq \tau$ there is a unique morphism $\omega \rightarrow \tau$, as there are no self-intersections of cells). If $e \in \mathscr{P}_{1}(\tau)$, $e: \omega \rightarrow \tau$, then we can calculate $\check{\Delta}_{e}(\tau)$ using Proposition 3.15. Choose some $\sigma, \sigma^{\prime} \in \mathscr{P} \tilde{\Sigma}_{\nabla}^{\prime}$ maximal containing $\tau$, and let $v, v^{\prime}$ be the two endpoints of $\omega$. Write $\omega=\delta\left(\omega_{1}\right)+\omega_{2}$ with $C\left(\omega_{1} \times\{0\}\right)+C\left(\omega_{2} \times\{1\}\right) \in \tilde{\Sigma}^{\prime}, C\left(\omega_{1}\right) \in \Sigma^{\prime}$. By the MPCP assumption, $\omega_{1}$ is simplicial, with generators $n_{1}, \ldots, n_{k}, k \geq r$. If $k>r+1$, then $\operatorname{dim} \delta\left(\omega_{1}\right) \geq 2$, contradicting $\operatorname{dim} \omega=1$, so we have two cases. Either $\omega_{1}=\operatorname{Conv}\left\{n_{1}, \ldots, n_{r}\right\}$ with $\varphi_{i}\left(n_{j}\right)=\delta_{i j}$, or $\omega_{1}=\operatorname{Conv}\left\{n_{1}, \ldots, n_{r}, n_{p}^{\prime}\right\}$ with $\varphi_{i}\left(n_{j}\right)=\delta_{i j}$ and $\varphi_{p}\left(n_{p}^{\prime}\right)=1$ for some $p$. In the former case, it follows from Proposition 3.15 that there is no monodromy around the loop $\gamma$ determined by the data $v, v^{\prime}, \sigma, \sigma^{\prime}$. In the second case,

$$
v=n_{1}+\cdots+n_{r}+w, \quad v^{\prime}=n_{1}+\cdots+n_{p}^{\prime}+\cdots+n_{r}+w^{\prime}
$$


for $w, w^{\prime} \in \omega_{2}$, and

$$
T_{\gamma}(n)=n+\left\langle m_{p}^{\prime}-m_{p}, n\right\rangle\left(n_{p}^{\prime}-n_{p}\right) .
$$

Noting again by the MPCP assumption $n_{p}^{\prime}-n_{p}$ is primitive, and it follows that we can take, up to translation,

$$
\check{\Delta}_{e}(\tau)=\operatorname{Conv}\left\{m_{p} \mid \begin{array}{l}
\left(m_{p}, l_{p}\right) \text { defines }-\tilde{\varphi}_{p} \text { on the cone of } \tilde{\Sigma}^{\prime} \\
\text { corresponding to } \sigma, \tau \subseteq \sigma, \sigma \text { maximal }
\end{array}\right\} .
$$

This only depends on the index $p$, which depends only on $e$, so we write this index as $p_{e}$. Then we set for $1 \leq i \leq r$,

$$
\Omega_{i}=\left\{e: \omega \rightarrow \tau \mid \operatorname{dim} \omega_{1}=r+1, p_{e}=i\right\}
$$

and for $e \in \Omega_{i}$,

$$
\check{\Delta}_{e}(\tau)=\delta_{i}:=\operatorname{Conv}\left\{m_{i} \mid \begin{array}{l}
\left(m_{i}, l_{i}\right) \text { defines }-\tilde{\varphi}_{i} \text { on the cone of } \tilde{\Sigma}^{\prime} \\
\text { corresponding to } \sigma, \tau \subseteq \sigma, \sigma \text { maximal }
\end{array}\right\} .
$$

Now $\delta_{i}$ is the image under projection of a face of $\tilde{\Delta}_{i}$, determining the function $-\tilde{\varphi}_{i}$ on the cone of $\tilde{\Sigma}^{\prime}$ corresponding to $\tau$. It is then easy to see that if this cone is $C\left(\tau_{1} \times\right.$ $\{0\})+C\left(\tau_{2} \times\{1\}\right)$, and $\delta_{i}^{\prime}$ is the set of elements of $\Delta_{i}$ giving the value of $-\varphi_{i}$ on $\tau_{1}$ and $\delta^{\prime \prime}=N_{\nabla \check{h}^{\prime}}\left(\tau_{2}\right) \in \check{\Sigma}_{\check{h}^{\prime}}^{\prime}$, then $\delta_{i}=\delta_{i}^{\prime} \cap \delta^{\prime \prime}$. Then by Lemma 2.7, $\delta^{\prime}=\operatorname{Conv}\left(\delta_{1}^{\prime}, \ldots, \delta_{r}^{\prime}\right)$ is a face of $\nabla^{*}$ and also $\operatorname{Conv}\left(\delta_{1}, \ldots, \delta_{r}\right)=\delta^{\prime} \cap \delta^{\prime \prime}$, so the cone over $\operatorname{Conv}\left(\delta_{1}, \ldots, \delta_{r}\right)$ is a cone in $\check{\Sigma}^{\prime}$, hence $\operatorname{Conv}\left(\delta_{1}, \ldots, \delta_{r}\right)$ is an elementary simplex. From this one concludes that in $M_{\mathbb{R}} \times \mathbb{R}^{r}$, $\operatorname{Conv}\left(\delta_{1} \times\left\{e_{1}\right\}, \ldots, \delta_{r} \times\left\{e_{r}\right\}\right)$ is an elementary simplex with $e_{1}, \ldots, e_{r}$ the standard basis of $\mathbb{R}^{r}$. This is the first condition needed for simplicity. The second statement is essentially dual. Taking $f \in \mathscr{P}_{n-r-1}(\tau)$, a similar argument shows that

$$
\Delta_{f}(\tau)=\beta_{i}^{*}\left(\tau_{1}\right)
$$

for some $i$ and the cone over $\tau_{1}=\operatorname{Conv}\left(\beta_{1}^{*}\left(\tau_{1}\right), \ldots, \beta_{r}^{*}\left(\tau_{1}\right)\right)$ is a cone in $\Sigma^{\prime}$, so by the MPCP assumption we conclude $\operatorname{Conv}\left(\beta_{1}^{*}\left(\tau_{1}\right) \times\left\{e_{1}\right\}, \ldots, \beta_{r}^{*}\left(\tau_{r}\right) \times\left\{e_{r}\right\}\right)$ is an elementary simplex.

We now wish to discuss duality. To do so, we need to polarize the degeneration $f$ : $\mathcal{X}^{\prime} \rightarrow \mathcal{S}$, and we do this by suitably polarizing the ambient space $X\left(\tilde{\Sigma}^{\prime}\right)$. We will use the following:

Definition 3.17. A subdivision $\tilde{\Sigma}^{\prime}$ of $\tilde{\Sigma}$ with an integral piecewise linear function $\tilde{h}$ on $\tilde{\Sigma}^{\prime}$ is good for $\Sigma^{\prime}, h$ if $\tilde{\Sigma}^{\prime}$ is a good subdivision of $\tilde{\Sigma}$ (Definition 3.8), $\left.\tilde{h}\right|_{N_{\mathbb{R}} \times\{0\}}=h, \tilde{h}$ is strictly convex, and $\tilde{h}^{\prime}:=\tilde{h}-\tilde{\varphi}$ is convex on $\tilde{\Sigma}^{\prime}$. 
Remark 3.18. If $\tilde{\Sigma}^{\prime}, \tilde{h}$ is good for $\Sigma^{\prime}, h$, then $\tilde{h}$ and $\tilde{\Sigma}^{\prime}$ are completely determined by $h$ and the values of $\tilde{h}$ on $C\left(\nabla^{h^{\prime}} \times\{1\}\right)$. Indeed, $\tilde{\Sigma}^{\prime}$ is a subdivision of $\tilde{\Sigma}$, and all one-dimensional cones of $\tilde{\Sigma}^{\prime}$ are either one-dimensional cones of $\Sigma^{\prime}$ or contained in $C\left(\nabla^{\check{h}^{\prime}} \times\{1\}\right)$. Thus $\tilde{\Sigma}^{\prime}$ is the unique such coherent subdivision on which the given values of $\tilde{h}$ extend to a convex piecewise linear function on $\tilde{\Sigma}^{\prime}$. More precisely, if we have a cone $\rho=C(\sigma) \times\{0\}+C(\tau \times\{1\})$ of $\tilde{\Sigma}$ with $\sigma$ a face of $\Delta^{*}, \tau$ a face of $\nabla^{\breve{h}^{\prime}}$, the values of $\tilde{h}$ are given on $C(\sigma) \times\{0\}$ and $C(\tau \times\{1\})$. Let

$$
\tilde{\rho}=\operatorname{Conv}(\{(n, 0, h(n)) \mid n \in C(\sigma)\} \cup\{(n, l, \tilde{h}(n, l)) \mid(n, l) \in C(\tau \times\{1\})\}) .
$$

Then $\tilde{h}$ can be defined by, for $(n, l) \in \rho$,

$$
\tilde{h}(n, l)=\inf \{r \mid(n, l, r) \in \tilde{\rho}\} .
$$

Of course, $\tilde{h}$ is strictly convex piecewise linear on a coherent subdivision of $\rho$ which does not involve any one-dimensional rays not in $C(\sigma) \times\{0\}$ or $C(\tau \times\{1\})$, and this is the unique such extension given the values on $C(\sigma)$ and $C(\tau \times\{1\})$.

One should note that an arbitrary choice of values for $\tilde{h}$ on $C\left(\nabla^{\check{h}^{\prime}} \times\{1\}\right)$ does not necessarily yield a function $\tilde{h}$ which is strictly convex on the whole fan $\tilde{\Sigma}^{\prime}$, and this can make it difficult, in general, to find good choices for $\tilde{h}$.

All of the above story can be repeated, interchanging the roles of $\Delta$ and $\nabla, h$ and $\check{h}$, so we get fans $\widetilde{\Sigma}$ and the notion of good subdivisions $\widetilde{\Sigma}^{\prime}$, etc.

Now since the choice of $\tilde{\Sigma}^{\prime}, \tilde{h}, \widetilde{\Sigma^{\prime}}, \widetilde{\breve{h}}$ are not unique (even their existence hasn't been addressed yet, see the end of this section), there is no reason for the corresponding dual intersection complexes $\left(B_{\nabla}^{\check{h}}, \mathscr{P} \tilde{\Sigma}_{\nabla}^{\prime \prime}\right)$ and $\left(B_{\Delta}^{h}, \mathscr{P}_{\Delta}^{\widetilde{\Sigma^{\prime}}}\right)$ to be related by a discrete Legendre transform. However, we will show for any good choice of $\tilde{\Sigma}^{\prime}, \tilde{h}$, we can find the correct $\widetilde{\Sigma^{\prime}}$, $\widetilde{h}$ which will yield dual intersection complexes related by a discrete Legendre transform. This will be done essentially via a Legendre transform in $N_{\mathbb{R}}$.

Suppose we are given $\tilde{\Sigma}^{\prime}, \tilde{h}$ good for $\Sigma^{\prime}, h$. We have

$$
\tilde{\nabla}=\left\{(n, l) \mid n \in \nabla, l \geq h^{\prime}(n)\right\} \subseteq N_{\mathbb{R}} \oplus \mathbb{R} .
$$

Set

$$
\tilde{\nabla}^{\prime}=\left\{(n, l) \mid n \in \nabla^{\check{h}^{\prime}}, l \geq \tilde{h}^{\prime}(n, 1)\right\} \subseteq N_{\mathbb{R}} \oplus \mathbb{R} .
$$

Let $\widetilde{\Sigma^{\prime}}$ be the normal fan to $\tilde{\nabla}+\tilde{\nabla}^{\prime}$ in $M_{\mathbb{R}} \oplus \mathbb{R}$, and set

$$
\widetilde{h}(m, l)=-\inf \left\{\left\langle(m, l),\left(n, l^{\prime}\right)\right\rangle \mid\left(n, l^{\prime}\right) \in \tilde{\nabla}+\tilde{\nabla}^{\prime}\right\}
$$

to be the piecewise linear function on $\widetilde{\check{\Sigma}^{\prime}}$ defined by $\tilde{\nabla}+\tilde{\nabla}^{\prime}$. Let $\widetilde{h^{\prime}}=\widetilde{\breve{h}}-\widetilde{\varphi}$, so

$$
\widetilde{\tilde{h}^{\prime}}(m, l)=-\inf \left\{\left\langle(m, l),\left(n, l^{\prime}\right)\right\rangle \mid\left(n, l^{\prime}\right) \in \tilde{\nabla}^{\prime}\right\} .
$$


Set

$$
\tilde{\Delta}^{\prime}=\left\{(m, l) \mid m \in \Delta^{h^{\prime}}, l \geq \widetilde{\tilde{h}^{\prime}}(m, 1)\right\} .
$$

We first wish to show that $\widetilde{\Sigma^{\prime}}, \widetilde{\breve{h}}$ is good for $\check{\Sigma}^{\prime}, \check{h}$ and that this setup is dual, i.e. $\tilde{\Sigma}^{\prime}, \tilde{h}$ can be recovered from $\widetilde{\Sigma^{\prime}}, \widetilde{\breve{h}}$ in the same manner. To this end,

Lemma 3.19.

$$
\tilde{\nabla}+\tilde{\nabla}^{\prime}=\left\{(n, l) \mid n \in \nabla^{h} \text { and } l \geq \tilde{h}^{\prime}(n, 1)\right\} .
$$

Proof. First we need

Claim 3.20. Let $C(\sigma) \times\{0\}+C(\tau \times\{1\})$ be a cone of $\tilde{\Sigma}^{\prime}, \sigma \subseteq \partial \Delta^{*}, \tau \subseteq \partial \nabla^{\check{h}^{\prime}}$. Then

$$
(C(\sigma) \cap \nabla)+\tau=(C(\sigma)+\tau) \cap \nabla^{\check{h}} .
$$

Proof. Inclusion of the first set in the second is obvious. Conversely, as in the proof of Claim 3.6, it is enough to show that if $\rho$ is a proper face of $(C(\sigma) \cap \nabla)+\tau$ not contained in $\partial(C(\sigma)+\tau)$, then $\rho \subseteq \partial \nabla^{\breve{h}}$. Let $\rho_{i}=\beta_{i}^{*}(\sigma), \sigma_{i}=\operatorname{Conv}\left\{0, \rho_{i}\right\}$. By Claim 3.6, $C(\sigma) \cap \nabla=$ $\sigma_{1}+\cdots+\sigma_{r}$. Furthermore, if $\rho$ is a face as above, then there is some $i$ such that

$$
\rho \subseteq \sigma_{1}+\cdots+\rho_{i}+\cdots+\sigma_{r}+\tau .
$$

Now $\sigma \subseteq \partial \Delta^{*}$, so there exists a face $\omega \subseteq \partial \Delta$ dual to the minimal face of $\Delta^{*}$ containing $\sigma$, and there exists a cone $\tau^{\prime} \in \check{\Sigma}_{\check{h}^{\prime}}^{\prime}$ corresponding to the minimal face of $\nabla^{\check{h}^{\prime}}$ containing $\tau$. By Lemma 3.1, $\omega \cap \tau^{\prime} \neq \emptyset$. In particular, there is a subcone $\tau^{\prime \prime} \in \check{\Sigma}^{\prime}$ of $\tau^{\prime}$ with $\omega \cap \tau^{\prime \prime} \neq \emptyset$. Let $m \in \omega \cap \tau^{\prime \prime}$. It follows from Claim 3.6 that $m=m_{1}+\cdots+m_{r}$ for $m_{j} \in \tau^{\prime \prime} \cap \Delta_{j}$, $\left\langle m_{i}, \sigma_{j}\right\rangle=0$ for $i \neq j,\left\langle m_{i}, \rho_{i}\right\rangle=-1$, and $\left\langle m_{i}, \tau\right\rangle=-\check{h}^{\prime}\left(m_{i}\right)$. Thus

$$
\left\langle m_{i}, \rho\right\rangle=-1-\check{h}^{\prime}\left(m_{i}\right)=-\check{h}\left(m_{i}\right) .
$$

Thus we conclude that $\rho \subseteq \partial \nabla^{\check{h}}$.

Now define $\bar{h}: \nabla^{h} \rightarrow \mathbb{R}$ by

$$
\bar{h}(n)=\min \left\{l \mid(n, l) \in \tilde{\nabla}+\tilde{\nabla}^{\prime}\right\} .
$$

Then

$$
\bar{h}(n)=\min \left\{h^{\prime}\left(n_{1}\right)+\tilde{h}^{\prime}\left(n_{2}, 1\right) \mid n_{1} \in \nabla, n_{2} \in \nabla^{\check{h}^{\prime}}, n_{1}+n_{2}=n\right\} .
$$

By convexity of $\tilde{h}^{\prime}, \bar{h}(n) \geq \tilde{h}^{\prime}(n, 1)$. If $n \in \nabla^{\check{h}^{\prime}}$, then

$$
\bar{h}(n) \leq h^{\prime}(0)+\tilde{h}^{\prime}(n, 1)=\tilde{h}^{\prime}(n, 1),
$$

so $\bar{h}=\tilde{h}^{\prime}(\cdot, 1)$ on $\nabla^{\check{h}^{\prime}}$. On the other hand, if $n \in \nabla^{\check{h}} \backslash \nabla^{\check{h}^{\prime}}$, then $(n, 1)$ is contained in a cone $C(\sigma) \times\{0\}+C(\tau \times\{1\})$, and thus we can write, by the claim, $n=n_{1}+n_{2}$ for $n_{1} \in C(\sigma) \cap \nabla, n_{2} \in \tau$. Then since $\tilde{h}^{\prime}$ is linear on this cone,

$$
\bar{h}(n) \leq h^{\prime}\left(n_{1}\right)+\tilde{h}^{\prime}\left(n_{2}, 1\right)=\tilde{h}^{\prime}(n, 1) .
$$

Thus $\bar{h}=\tilde{h}^{\prime}(\cdot, 1)$ on $\nabla^{\check{h}}$, as desired. 
Theorem 3.21. $\widetilde{\Sigma^{\prime}}$ and $\widetilde{\breve{h}}$ are good for $\check{\Sigma}^{\prime}, \check{h}$.

Proof. Because $\widetilde{\Sigma}$ is the normal fan to $\tilde{\nabla}, \widetilde{\Sigma^{\prime}}$ is a subdivision of $\widetilde{\Sigma}$. To check that it is good for $\check{\Sigma}^{\prime}$, we need to consider normal vectors to maximal lower faces of $\tilde{\nabla}+\tilde{\nabla}^{\prime}$. Such a face is a graph of $\tilde{h}^{\prime}(\cdot, 1)$ over its projection to $N_{\mathbb{R}}$ by Lemma 3.19. Let $\rho \in \tilde{\Sigma}^{\prime}$ be a maximal cone, with $-\tilde{h}^{\prime}$ given by $(m, l) \in M \oplus \mathbb{Z}$, and let

$$
\tilde{\rho}=\left\{\left(n, \tilde{h}^{\prime}(n, 1)\right) \mid(n, 1) \in \rho \cap\left(\nabla^{\check{h}} \times\{1\}\right)\right\},
$$

so that $\tilde{\rho}$ is contained in a maximal lower face of $\tilde{\Delta}+\tilde{\Delta}^{\prime}$. The primitive normal vector to this face is then $(m, 1)$, so we only need to check that $m \in \Delta^{h^{\prime}}$. But by convexity of $\tilde{h}^{\prime}$,

$$
\langle m, n\rangle=\langle(m, l),(n, 0)\rangle \geq-\tilde{h}^{\prime}(n, 0)=-h^{\prime}(n)
$$

for all $n \in N_{\mathbb{R}}$, so $m \in \Delta^{h^{\prime}}$.

The next result shows that by applying this procedure to $\widetilde{\Sigma^{\prime}}$ and $\widetilde{h}$, we get back to $\tilde{\Sigma}^{\prime}$, $\tilde{h}$.

\section{Theorem 3.22.}

$$
\tilde{h}^{\prime}(n, l)=-\inf \left\{\left\langle\left(m, l^{\prime}\right),(n, l)\right\rangle \mid\left(m, l^{\prime}\right) \in \tilde{\Delta}^{\prime}\right\}
$$

and

$$
\tilde{h}(n, l)=-\inf \left\{\left\langle\left(m, l^{\prime}\right),(n, l)\right\rangle \mid\left(m, l^{\prime}\right) \in \tilde{\Delta}+\tilde{\Delta}^{\prime}\right\}
$$

for any $(n, l)$ in the support of the fan $\tilde{\Sigma}^{\prime}$.

Proof. By the dual of Theorem 3.21, $\tilde{\Delta}+\tilde{\Delta}^{\prime}$ induces a good subdivision $\tilde{\Sigma}_{1}^{\prime}$, $\tilde{h}_{1}$ for $\Sigma^{\prime}, h$. We just need to show $\tilde{h}_{1}=\tilde{h}$, but by Remark 3.18, it is enough to check this on $C\left(\nabla^{\check{h}^{\prime}} \times\{1\}\right)$. But $\tilde{h}=\tilde{h}^{\prime}$ on $C\left(\nabla^{\check{h}^{\prime}} \times\{1\}\right)$, so it is enough to check the first equality on $\nabla^{\check{h}^{\prime}} \times\{1\}$.

Let $n \in \nabla^{\check{h}^{\prime}},(m, l) \in \tilde{\Delta}^{\prime}$. Then

$$
\begin{aligned}
\langle(m, l),(n, 1)\rangle & =\langle m, n\rangle+l \\
& \geq\langle m, n\rangle+\widetilde{h^{\prime}}(m, 1) \\
& =\langle m, n\rangle-\inf \left\{\left\langle(m, 1),\left(n^{\prime}, l^{\prime}\right)\right\rangle \mid\left(n^{\prime}, l^{\prime}\right) \in \tilde{\nabla}^{\prime}\right\} \\
& \geq\langle m, n\rangle-\left\langle(m, 1),\left(n, \tilde{h}^{\prime}(n, 1)\right)\right\rangle \\
& =-\tilde{h}^{\prime}(n, 1) .
\end{aligned}
$$

Thus

$$
\tilde{h}^{\prime}(n, 1) \geq-\inf \left\{\langle(m, l),(n, 1)\rangle \mid(m, l) \in \tilde{\nabla}^{\prime}\right\} .
$$


Conversely, suppose $n \in \nabla^{\check{h}^{\prime}}$ is such that $(n, 1)$ is in a maximal cone of $\tilde{\Sigma}^{\prime}$ contained in $C\left(\nabla^{\check{h}^{\prime}} \times\{1\}\right)$. Then on this cone $-\tilde{h}^{\prime}$ is given by some $(m, l) \in M_{\mathbb{R}} \oplus \mathbb{R}$, so $l=$ $-\tilde{h}^{\prime}(n, 1)-\langle m, n\rangle$, and

$$
\begin{aligned}
\tilde{h^{\prime}}(m, 1) & =-\inf \left\{\left\langle(m, 1),\left(n^{\prime}, l^{\prime}\right)\right\rangle \mid\left(n^{\prime}, l^{\prime}\right) \in \tilde{\nabla}^{\prime}\right\} \\
& =-\inf \left\{\left\langle(m, 1),\left(n^{\prime}, \tilde{h}^{\prime}\left(n^{\prime}, 1\right)\right)\right\rangle \mid n^{\prime} \in \nabla^{\check{h}^{\prime}}\right\} \\
& =-\inf \left\{\left\langle m, n^{\prime}\right\rangle+\tilde{h}^{\prime}\left(n^{\prime}, 1\right) \mid n^{\prime} \in \nabla^{\check{h}^{\prime}}\right\} .
\end{aligned}
$$

By convexity of $\tilde{h}^{\prime}$, this infinum occurs when $n^{\prime}=n$, so $\widetilde{h^{\prime}}(m, 1)=l$. On the other hand, as observed in the proof of Theorem 3.21, $m \in \Delta^{h^{\prime}}$. Thus $(m, l) \in \tilde{\Delta}^{\prime}$ and

$$
-\inf \left\{\left\langle\left(m^{\prime}, l^{\prime}\right),(n, 1)\right\rangle \mid\left(m^{\prime}, l^{\prime}\right) \in \tilde{\nabla}^{\prime}\right\} \geq-\langle(m, l),(n, 1)\rangle=\tilde{h}^{\prime}(n, 1) .
$$

Thus equality holds.

Thus we have a way of passing between good data $\tilde{\Sigma}^{\prime}, \tilde{h}$ and good data $\widetilde{\Sigma^{\prime}}, \widetilde{h}$, and this procedure is reversible. To complete the story, we need a method of passing between relevant cones of $\tilde{\Sigma}^{\prime}$ and relevant cones of $\widetilde{\Sigma^{\prime}}$.

Let

$$
\rho=C(\sigma) \times\{0\}+C(\tau \times\{1\})
$$

be a relevant cone of $\tilde{\Sigma}^{\prime}$. Then $\lambda(\rho):=\ell(\sigma)+\tau$ is contained in a face of $\nabla^{h}$. Let

$$
\begin{aligned}
\tilde{\lambda}(\rho) & :=\left\{\left(n, h^{\prime}(n)\right) \mid n \in \delta(\sigma)\right\}+\left\{\left(n, \tilde{h}^{\prime}(n, 1)\right) \mid n \in \tau\right\} \\
& =\left\{\left(n, \tilde{h}^{\prime}(n, 1)\right) \mid n \in \lambda(\rho)\right\},
\end{aligned}
$$

the last equality following from $\tilde{h}^{\prime}$ being linear on $\rho$. By Lemma 3.19, $\tilde{\lambda}(\rho) \subseteq \tilde{\nabla}+\tilde{\nabla}^{\prime}$. Let

$$
\alpha(\rho)=N_{\tilde{\nabla}+\tilde{\nabla}^{\prime}}(\tilde{\lambda}(\rho)) .
$$

Then $\alpha(\rho)$ is a cone in $\widetilde{\Sigma}^{\prime}$. We can similarly, for a relevant cone $\check{\rho}$ of $\widetilde{\Sigma}^{\prime}$, define $\check{\lambda}(\check{\rho}), \widetilde{\grave{\lambda}}(\check{\rho})$, and a cone $\check{\alpha}(\check{\rho})$ of $\tilde{\Sigma}^{\prime}$.

Proposition 3.23. If $\rho$ is a relevant cone of $\tilde{\Sigma}^{\prime}$, then $\tilde{\lambda}(\rho)$ is a face of $\tilde{\nabla}+\tilde{\nabla}^{\prime}$ and $\alpha(\rho)$ is a relevant cone of $\widetilde{\Sigma}$.

Proof. First observe that $\alpha(\rho)$ is a cone of the second type of Observation 3.9. To see this, just observe that $\tilde{h}^{\prime}(\cdot, 1)$ is affine linear on $\delta(\sigma)+\tau$ and $\delta(\sigma)+\tau$ is contained in $\partial \nabla^{\check{h}}$, so by Lemma 3.19, $\tilde{\lambda}(\rho)$ is contained in a face of $\tilde{\nabla}+\tilde{\nabla}^{\prime}$ projecting injectively into $\partial \nabla^{\breve{h}}$. Then $\alpha(\rho)$ is clearly of the second type.

Note now that the faces of $\tilde{\nabla}+\tilde{\nabla}^{\prime}$ whose normal cones are of the second type are in one-to-one correspondence via projection $\check{p}: N_{\mathbb{R}} \oplus \mathbb{R} \rightarrow N_{\mathbb{R}}$ with the collection of subsets of $\partial \nabla^{\check{h}}$ given by

$$
\left\{\check{p}((\omega \times\{1\}) \cap \delta) \mid \omega \text { is a face of } \nabla^{\check{h}} \text { and } \delta \text { is a normal cone to } \tilde{\Delta}^{\prime}\right\} .
$$


Indeed, the function $\tilde{h}^{\prime}$ is linear on normal cones of $\tilde{\Delta}^{\prime}$ by Theorem 3.22, and the statement then follows from Lemma 3.19. So to show $\tilde{\lambda}(\rho)$ is a face of $\tilde{\nabla}+\tilde{\nabla}^{\prime}$ we need to show $\lambda(\rho)=\check{p}((\omega \times\{1\}) \cap \delta)$ for some face $\omega$ of $\nabla^{\check{h}}$ and $\delta$ a normal cone of $\tilde{\Delta}^{\prime}$. But as $\rho$ is a normal cone to a face of $\tilde{\Delta}+\tilde{\Delta}^{\prime}$, it is necessarily of the form $\rho^{\prime} \cap \delta$, where $\rho^{\prime}$ is a normal cone to $\tilde{\Delta}$, i.e. $\rho^{\prime} \in \tilde{\Sigma}$, and $\delta$ is a normal cone to $\tilde{\Delta}^{\prime}$. Then $\rho^{\prime}=C\left(\sigma^{\prime}\right) \times\{0\}+C\left(\tau^{\prime} \times\{1\}\right)$ with $\sigma^{\prime}+\tau^{\prime}$ a face of $\Delta^{*}+\nabla^{h^{\prime}}$, and $\delta\left(\sigma^{\prime}\right)+\tau^{\prime}=: \omega$ is a face of $\nabla+\nabla^{\check{h}^{\prime}}=\nabla^{\check{h}}$. Using the notation of the proof of Theorem 3.10, we can take $V_{\rho}=V_{\rho^{\prime}}$, and then by Claim 3.11,

$$
\begin{aligned}
\lambda(\rho) \times\{1\} & =(\delta(\sigma)+\tau) \times\{1\} \\
& =V_{\rho}^{\perp} \cap \rho \cap\left(N_{\mathbb{R}} \times\{1\}\right) \\
& =V_{\rho}^{\perp} \cap \rho^{\prime} \cap \delta \cap\left(N_{\mathbb{R}} \times\{1\}\right) \\
& =(\omega \times\{1\}) \cap \delta,
\end{aligned}
$$

as desired.

Next write $\check{\rho}=\alpha(\rho)$,

$$
\check{\rho}=C(\check{\sigma}) \times\{0\}+C(\check{\tau} \times\{1\}) .
$$

To show $\check{\rho}$ is relevant, we show $\beta(\check{\sigma}) \neq \emptyset$. Now $\rho$ is of course contained in $\rho^{\prime} \in \tilde{\Sigma}$, so $\sigma \subseteq \sigma^{\prime}$ and $\tau \subseteq \tau^{\prime}$. Changing the meaning of $\omega$, for notational convenience, write $\omega^{*}:=\sigma^{\prime}$, so we can write $\partial\left(\sigma^{\prime}\right)=m$, and write $\omega, m^{*}$ as in $\S 2$. Then $\delta(\sigma) \subseteq m$.

Now $C(\check{\sigma})=N_{\nabla \check{h}}(g(\sigma)+\tau)$, and as $\nabla^{\check{h}}=\nabla+\nabla^{\check{h}^{\prime}}$,

$$
C(\check{\sigma})=N_{\nabla}(\delta(\sigma)) \cap N_{\nabla^{\prime}{ }^{\prime}}(\tau)
$$

$C\left(m^{*}\right)=N_{\nabla}(m) \subseteq N_{\nabla}(g(\sigma))$, and $N_{\nabla_{h^{\prime}}}\left(\tau^{\prime}\right) \subseteq N_{\nabla_{h^{\prime}}}(\tau)$. On the other hand, $\omega=\beta\left(m^{*}\right)$ is the dual face of $\Delta$ to $\omega^{*}$ by Lemma 2.7 , and $\omega^{*}+\tau^{\prime}$ is a face of $\Delta^{*}+\nabla^{\check{h}^{\prime}}$ by Proposition 3.7. Thus

$$
\begin{aligned}
\emptyset \neq N_{\Delta^{*}+\nabla^{h^{\prime}}}\left(\omega^{*}+\tau^{\prime}\right) & =N_{\Delta^{*}}\left(\omega^{*}\right) \cap N_{\nabla_{\check{h}^{\prime}}}\left(\tau^{\prime}\right) \\
& =C(\omega) \cap N_{\nabla^{h^{\prime}}}\left(\tau^{\prime}\right) .
\end{aligned}
$$

Since $C(\omega) \subseteq C\left(m^{*}\right)$ and $\check{\varphi}_{i}$ is non-zero at each non-zero point of $C(\omega)$, it follows that for each $i$, there exists a point in $C\left(m^{*}\right) \cap N_{\nabla_{\check{h}^{\prime}}}\left(\tau^{\prime}\right)$ on which $\check{\varphi}_{i}$ is non-zero. Thus in particular, as $m^{*} \cap N_{\nabla^{\prime} h^{\prime}}\left(\tau^{\prime}\right)$ has integral vertices, $\delta\left(m^{*} \cap N_{\nabla_{h^{\prime}}}\left(\tau^{\prime}\right)\right) \neq \emptyset$. But $m^{*} \cap N_{\nabla_{\check{h}^{\prime}}}\left(\tau^{\prime}\right) \subseteq \check{\sigma}$, so $\beta(\check{\sigma}) \neq \emptyset$.

Proposition 3.24. $\alpha$ and $\check{\alpha}$ give a one-to-one order reversing correspondence between relevant cones of $\tilde{\Sigma}^{\prime}$ and relevant cones of $\widetilde{\Sigma^{\prime}}$.

Proof. We need to show that $\rho=\check{\alpha}(\alpha(\rho))$. First, we show

$$
\rho \subseteq \check{\alpha}(\alpha(\rho))=N_{\tilde{\Delta}+\tilde{\Delta}^{\prime}}(\widetilde{\widetilde{\lambda}}(\alpha(\rho))) .
$$


Write $\rho=C(\sigma) \times\{0\}+C(\tau \times\{1\}), \check{\rho}=\alpha(\rho)$. Let $n \in \beta_{i}^{*}(\sigma) \subseteq \nabla_{i}$. Then $\left(n, h^{\prime}(n)\right)$ coincides with the function $-\widetilde{\varphi}_{i}$ on $\check{\rho}=C(\check{\sigma}) \times\{0\}+C(\check{\tau} \times\{1\})$. Now if $m_{1} \in \beta(\check{\sigma})$, $-\widetilde{\varphi}_{i}\left(m_{1}, 0\right)=-1$, and if $m_{2} \in \check{\tau},-\widetilde{\varphi}_{i}\left(m_{2}, 1\right)=0$. Thus

$$
\begin{aligned}
\left\langle(n, 0),\left(m_{1}, \check{h}^{\prime}\left(m_{1}\right)\right)+\left(m_{2}, \widetilde{h^{\prime}}\left(m_{2}, 1\right)\right)\right\rangle & =\left\langle\left(n, h^{\prime}(n)\right),\left(m_{1}, 0\right)+\left(m_{2}, 1\right)\right\rangle-h^{\prime}(n) \\
& =-1-h^{\prime}(n),
\end{aligned}
$$

so $(n, 0)$ is constant on $\widetilde{\bar{\lambda}}(\alpha(\rho))$. If on the other hand $\left(m_{1}, l_{1}\right) \in \tilde{\Delta},\left(m_{2}, l_{2}\right) \in \tilde{\Delta}^{\prime}$, then

$$
\begin{aligned}
\left\langle(n, 0),\left(m_{1}, l_{1}\right)+\left(m_{2}, l_{2}\right)\right\rangle & =\left\langle\left(n, h^{\prime}(n)\right),\left(m_{1}, 0\right)+\left(m_{2}, 1\right)\right\rangle-h^{\prime}(n) \\
& \geq-\widetilde{\varphi}_{i}\left(m_{1}, 0\right)-\widetilde{\varphi}_{i}\left(m_{2}, 1\right)-h^{\prime}(n) \\
& \geq-1-h^{\prime}(n)
\end{aligned}
$$

Thus $(n, 0) \in N_{\tilde{\Delta}+\tilde{\Delta}^{\prime}}(\widetilde{\grave{\lambda}}(\alpha(\rho)))$. Just as in the proof of Lemma $2.7, \sigma=\operatorname{Conv}\left\{\beta_{1}^{*}(\sigma), \ldots, \beta_{r}^{*}(\sigma)\right\}$ so $C(\sigma) \times\{0\} \subseteq \check{\alpha}(\alpha(\rho))$.

Next let $n \in \tau$. Consider $\left(m_{1}, l_{1}\right) \in \tilde{\Delta},\left(m_{2}, l_{2}\right) \in \tilde{\Delta}^{\prime}$. Now by Theorem 3.22,

$$
\left\langle(n, 1),\left(m_{1}, l_{1}\right)+\left(m_{2}, l_{2}\right)\right\rangle \geq-\tilde{h}^{\prime}(n, 1) .
$$

On the other hand, by the definition of $\widetilde{h},\left(n, \tilde{h}^{\prime}(n, 1)\right)$ defines the function $-\tilde{h}^{\prime}$ on $\check{\rho}$, so if $m_{1} \in \beta(\check{\sigma}), m_{2} \in \check{\tau}$,

$$
\left\langle\left(n, \tilde{h}^{\prime}(n, 1)\right),\left(m_{1}, 0\right)+\left(m_{2}, 1\right)\right\rangle=-\tilde{h}^{\prime}\left(m_{1}+m_{2}, 1\right)
$$

or

$$
\left\langle n, m_{1}+m_{2}\right\rangle+\widetilde{\tilde{h}^{\prime}}\left(m_{1}+m_{2}, 1\right)=-\tilde{h}^{\prime}(n, 1)
$$

or

$$
\left\langle(n, 1),\left(m_{1}, \check{h}^{\prime}\left(m_{1}\right)\right)+\left(m_{2}, \widetilde{\tilde{h}^{\prime}}\left(m_{2}, 1\right)\right)\right\rangle=-\tilde{h}^{\prime}(n, 1) .
$$

Thus $(n, 1)$ takes the constant value $-\tilde{h}^{\prime}(n, 1)$ on $\widetilde{\grave{\lambda}}(\alpha(\rho))$, so $C(\tau \times\{1\}) \subseteq \check{\alpha}(\alpha(\rho))$. Thus $\rho \subseteq \check{\alpha}(\alpha(\rho))$.

To show equality, we compute dimensions. Let $n=\operatorname{dim} M_{\mathbb{R}}$. Then if $\operatorname{dim} \rho=k, \rho$ corresponds to an $n+1-k$ dimensional stratum of $X\left(\tilde{\Sigma}^{\prime}\right)$, and hence $\lambda(\rho)$ is an $(n-r)-$ $(n+1-k)$ dimensional cell of $\mathscr{P} \tilde{\Sigma}_{\nabla}^{\prime}$, and $\widetilde{\lambda}(\rho)$ is the same dimension. By Proposition 3.23, $\widetilde{\lambda}(\rho)$ is a face of $\tilde{\nabla}+\tilde{\nabla}^{\prime}$, so $\operatorname{dim} \alpha(\rho)=(n+1)-[(n-r)-(n+1-k)]=n-k+r+2$. Then $\operatorname{dim} \check{\alpha}(\alpha(\rho))=n-(n-k+r+2)+r+2=k$, so $\rho=\check{\alpha}(\alpha(\rho))$.

The functions $\tilde{h}$ and $\widetilde{h}$ give ample divisors on $X\left(\tilde{\Sigma}^{\prime}\right)$ and $X\left(\widetilde{\Sigma^{\prime}}\right)$ respectively, and these restrict to polarizations on $\mathcal{X}^{\prime} \rightarrow \mathcal{S}$ and $\check{\mathcal{X}}^{\prime} \rightarrow \mathcal{S}$. These then give rise to multi-valued

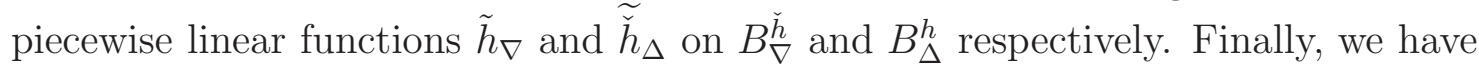

Theorem 3.25. $\left(B_{\Delta}, \mathscr{P}_{\Delta}^{\Sigma^{\prime}}, \widetilde{h}_{\Delta}\right)$ is the discrete Legendre transform of $\left(B_{\nabla}^{\check{h}}, \mathscr{P} \tilde{\Sigma}_{\nabla}^{\prime}, \tilde{h}_{\nabla}\right)$. 
Proof. By symmetry of the situation, it is enough to check that given a relevant cone $\rho$ of $\tilde{\Sigma}^{\prime}$, the piecewise linear function on the quotient fan $\tilde{\Sigma}^{\prime}(\rho)$ induced by $\tilde{h}$ has as Newton

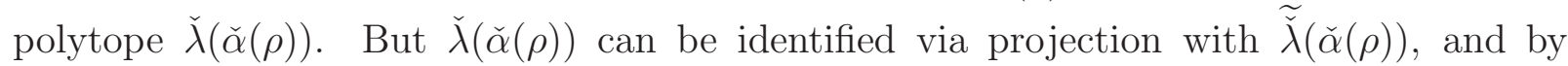
Theorem 3.22 , the latter is canonically identified with this Newton polytope.

Finally we discuss existence of pairs $\tilde{h}, \tilde{\Sigma}^{\prime} \operatorname{good}$ for $h, \Sigma^{\prime}$. Suppose that $h$ and $\breve{h}$ are given. Now I do not expect that in general one can find a $\tilde{h}, \tilde{\Sigma}^{\prime}$ good for $h, \Sigma^{\prime}$, but I do conjecture that one can find a pair $\tilde{h}, \tilde{\Sigma}^{\prime}$ good for $n h, \Sigma^{\prime}$, for $n$ a sufficiently large positive integer. This affects the affine structure on the intersection complex merely by rescaling by the factor $n$, so this is not a serious restriction. It arises due to integrality issues, as we will see below. However, this still seems to be a difficult combinatorial problem which has so far resisted attempts to solve. Thus we will settle for a weaker statement:

Theorem 3.26. There exists positive integers $n_{0}$ and $m_{0}$ such that for all $n \geq n_{0}$, there exists $\tilde{h}, \tilde{\Sigma}^{\prime}$ good for $m_{0} h+n \varphi, \Sigma$.

Proof. Begin by choosing the values of $\tilde{h}$ on $C\left(\nabla^{\check{h}^{\prime}} \times\{1\}\right)$. This can be done essentially arbitrarily; for convenience we take $\tilde{h} \equiv 0$ on this cone. In addition, we wish to take $\tilde{h}=h$ on $N_{\mathbb{R}} \times\{0\}$. Using Remark 3.18, each cone $\rho=C(\sigma) \times\{0\}+C(\tau \times\{1\})$ of $\tilde{\Sigma}$ can be subdivided in such a way that $\tilde{h}$ extends to a strictly convex piecewise linear function on this subdivision of $\rho$. These subdivisions are compatible: if $\rho \subseteq \rho^{\prime}$ and we subdivide $\rho$ and $\rho^{\prime}$ as in Remark 3.18, then the subdivision of $\rho^{\prime}$ restricts to the subdivision of $\rho$. Hence we obtain a subdivision $\tilde{\Sigma}^{\prime}$ of $\tilde{\Sigma}$ and a function $\tilde{h}$ on $\tilde{\Sigma}^{\prime}$. It is not necessarily integral, but does have rational slopes, so there exists $m_{0} \in \mathbb{Z}$ such that $m_{0} \tilde{h}$ is integral. Now strict convexity can be tested across codimension one cones of the fan $\tilde{\Sigma}^{\prime}$, and by construction the only cones which might cause problems are codimension one cones of $\tilde{\Sigma}^{\prime}$ contained in codimension one cones of $\tilde{\Sigma}$. But $\tilde{\varphi}$ is strictly convex on $\tilde{\Sigma}$, so there exists an $n_{0}$ such that $m_{0} \tilde{h}+n \tilde{\varphi}$ is convex on $\tilde{\Sigma}^{\prime}$ for all $n \geq n_{0}-1$ and strictly convex for $n \geq n_{0}$. Thus $m_{0} \tilde{h}+n \tilde{\varphi}$, $\tilde{\Sigma}^{\prime}$ is good for $m_{0} h+n \varphi, \Sigma^{\prime}$.

We note that as $h$ defines an ample divisor on $\mathbb{P}_{\Delta^{h}}, m_{0} h+n \varphi$ is also ample. Thus from the point of view of applying Theorem 0.1 to understand the topology of the SYZ fibration, this result is sufficient in the light of Theorem 3.16.

\section{An example, and COnnection to Kovalev's example}

We will consider Chad Schoen's example [15] of a Calabi-Yau threefold obtained as a fibred product of two rational elliptic surfaces. Let $f_{1}: Y_{1} \rightarrow \mathbb{P}^{1}$ and $f_{2}: Y_{2} \rightarrow \mathbb{P}^{1}$ be two rational elliptic surfaces with section, and suppose there is no point $x \in \mathbb{P}^{1}$ such that both $f_{1}^{-1}(x)$ and $f_{2}^{-1}(x)$ are singular curves. Then $X=Y_{1} \times_{\mathbb{P}^{1}} Y_{2}$ is a non-singular Calabi-Yau threefold with $\chi(X)=0$ and $h^{1,1}(X)=h^{1,2}(X)=19$. 
To study mirror symmetry for the Schoen fibred product, we proceed as in [8] by representing it as a complete intersection in $\mathbb{P}^{1} \times \mathbb{P}^{2} \times \mathbb{P}^{2}$ of hypersurfaces of tridegree $(1,3,0)$ and $(1,0,3)$. In terms of toric data, set

$$
\begin{aligned}
\bar{\Delta}_{1} & =[-1,1] \subseteq \mathbb{R} \\
\bar{\Delta}_{2}=\bar{\Delta}_{3} & =\operatorname{Conv}\{(-1,-1),(2,-1),(-1,2)\} \subseteq \mathbb{R}^{2} \\
\bar{\Delta}_{1}^{+} & =[0,1] \subseteq \mathbb{R} \\
\bar{\Delta}_{1}^{-} & =[-1,0] \subseteq \mathbb{R}
\end{aligned}
$$

and then

$$
\begin{aligned}
\Delta & =\bar{\Delta}_{1} \times \bar{\Delta}_{2} \times \bar{\Delta}_{3} \subseteq \mathbb{R} \times \mathbb{R}^{2} \times \mathbb{R}^{2}=M_{\mathbb{R}} \\
\Delta_{1} & =\bar{\Delta}_{1}^{+} \times \bar{\Delta}_{2} \times\{0\} \\
\Delta_{2} & =\bar{\Delta}_{1}^{-} \times\{0\} \times \bar{\Delta}_{3} .
\end{aligned}
$$

Label the vertices of $\Delta_{1}$ by

$$
\begin{array}{lll}
P_{0}^{0}=(0,-1,-1,0,0), & P_{1}^{0}=(0,2,-1,0,0), & P_{2}^{0}=(0,-1,2,0,0) \\
P_{0}^{+}=(1,-1,-1,0,0), & P_{1}^{+}=(1,2,-1,0,0), & P_{2}^{+}=(1,-1,2,0,0)
\end{array}
$$

and the vertices of $\Delta_{2}$ by

$$
\begin{array}{ccc}
Q_{0}^{0}=(0,0,0,-1,-1), & Q_{1}^{0}=(0,0,0,2,-1), & Q_{2}^{0}=(0,0,0,-1,2) \\
Q_{0}^{-}=(-1,0,0,-1,-1), & Q_{1}^{-}=(-1,0,0,2,-1), & Q_{2}^{-}=(-1,0,0,-1,2)
\end{array}
$$

so that

$$
\nabla^{*}=\operatorname{Conv}\left\{P_{i}^{0}, P_{i}^{+}, Q_{i}^{0}, Q_{i}^{-} \mid i=0,1,2\right\} .
$$

Setting $\bar{\Delta}_{1}^{*}=[-1,1] \subseteq \mathbb{R}$ and

$$
\bar{\Delta}_{2}^{*}=\bar{\Delta}_{3}^{*}=\operatorname{Conv}\{(-1,-1),(1,0),(0,1)\} \subseteq \mathbb{R}^{2},
$$

then one sees easily that

$$
\begin{aligned}
\Delta^{*} & =\operatorname{Conv}\left\{\bar{\Delta}_{1}^{*} \times\{0\} \times\{0\},\{0\} \times \bar{\Delta}_{2}^{*} \times\{0\},\{0\} \times\{0\} \times \bar{\Delta}_{3}^{*}\right\} \\
& \subseteq \mathbb{R} \times \mathbb{R}^{2} \times \mathbb{R}^{2}=N_{\mathbb{R}} .
\end{aligned}
$$

Let $R^{+}=(1,0,0,0,0)$ and $R^{-}=(-1,0,0,0,0)$,

$$
\begin{array}{lll}
S_{0}=(0,-1,-1,0,0), & S_{1}=(0,1,0,0,0), & S_{2}=(0,0,1,0,0) \\
T_{0}=(0,0,0,-1,-1), & T_{1}=(0,0,0,1,0), & T_{2}=(0,0,0,0,1)
\end{array}
$$

be points in $N_{\mathbb{R}}$. Then

$$
\begin{aligned}
& \nabla_{1}=\operatorname{Conv}\left\{0, R^{-}, S_{0}, S_{1}, S_{2}\right\} \\
& \nabla_{2}=\operatorname{Conv}\left\{0, R^{+}, T_{0}, T_{1}, T_{2}\right\}
\end{aligned}
$$


and one can compute that

$$
\nabla=\nabla_{1}+\nabla_{2}=\operatorname{Conv}\left\{R^{-}+T_{j}, R^{+}+S_{i}, S_{i}+T_{j} \mid i, j=0,1,2\right\} .
$$

Finally, we take $h=\varphi$ (corresponding to the anti-canonical polarization on $\mathbb{P}_{\Delta}=\mathbb{P}^{1} \times \mathbb{P}^{2} \times$ $\mathbb{P}^{2}$ ) and choose $\check{h}$ to give an ample divisor on a partial resolution of $\mathbb{P}_{\nabla}$. We will discuss the effect of this latter choice shortly. We can now describe $B_{\Delta^{h}}=B_{\Delta}$ as a polyhedral subcomplex of $\Delta$. There is one three-dimensional cell of $B_{\Delta}$ corresponding to each vertex of $\nabla$, and these are of the form (taking indices modulo 3 )

$$
\begin{aligned}
\sigma_{j}^{+} & =\operatorname{Conv}\left\{P_{0}^{+}, P_{1}^{+}, P_{2}^{+}\right\}+\operatorname{Conv}\left\{Q_{j+1}^{0}, Q_{j+2}^{0}\right\} \\
\sigma_{i}^{-} & =\operatorname{Conv}\left\{P_{i+1}^{0}, P_{i+2}^{0}\right\}+\operatorname{Conv}\left\{Q_{0}^{-}, Q_{1}^{-}, Q_{2}^{-}\right\} \\
\sigma_{i j} & =\operatorname{Conv}\left\{P_{i+1}^{0}, P_{i+2}^{0}, P_{i+1}^{+}, P_{i+2}^{+}\right\}+\operatorname{Conv}\left\{Q_{j+1}^{0}, Q_{j+2}^{0}, Q_{j+1}^{-}, Q_{j+2}^{-}\right\}
\end{aligned}
$$

corresponding to $R^{-}+T_{j}, S_{i}+R^{+}$and $S_{i}+T_{j}$ respectively. Then

$$
\begin{aligned}
& \bigcup_{j=0,1,2} \sigma_{j}^{+}=\{1\} \times \bar{\Delta}_{2} \times \partial \bar{\Delta}_{3} \\
& \bigcup_{i=0,1,2} \sigma_{i}^{-}=\{-1\} \times \partial \bar{\Delta}_{2} \times \bar{\Delta}_{3}
\end{aligned}
$$

are both solid tori, and

$$
\bigcup_{i, j=0,1,2} \sigma_{i j}=\bar{\Delta}_{1} \times \partial \bar{\Delta}_{2} \times \partial \bar{\Delta}_{3} .
$$

These three solids glue together to form an $S^{3}$ (this is just a variant of the usual Heegard splitting of the sphere).

We will describe the discriminant locus $\Gamma_{\Delta}$. In previous sections, we took the discriminant locus $\Gamma$ to be pretty large, including all codimension two simplices of $\operatorname{Bar}\left(\widetilde{\mathscr{P}} \underset{\Delta}{\widetilde{\Sigma^{\prime}}}\right)$ not containing vertices of $\mathscr{P} \underset{\Delta}{\widetilde{\Sigma^{\prime}}}$ or intersecting interiors of maximal cells. However, by Proposition 1.27 of [4], the affine structure can be extended across any simplex of $\Gamma$ for which the monodromy about this simplex is trivial. Thus, by applying Proposition 3.15, we can identify the minimal discriminant locus. This will depend on the choice of $\check{h}$, which we do not wish to specify precisely. However, we can specify the decomposition of $\nabla^{*}$ it induces, or less specifically, the decomposition of certain faces of $\nabla^{*}$, which will be enough for us.

Look first at $\sigma_{i}^{+}$. We can take, for example, the polyhedral decompositions $\mathscr{P}_{1}$ and $\mathscr{P}_{2}$ of $\{1\} \times \bar{\Delta}_{2} \times\{0\}$ and $\operatorname{Conv}\left\{Q_{j+1}^{0}, Q_{j+2}^{0}\right\}$ depicted here:
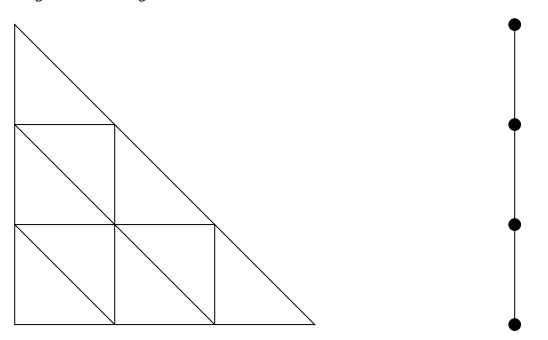
Then we obtain a polyhedral decomposition of the face of $\nabla^{*}$ dual to $R^{-}+T_{j}$ (this face is $\left.\operatorname{Conv}\left\{P_{0}^{+}, P_{1}^{+}, P_{2}^{+}, Q_{j+1}^{0}, Q_{j+2}^{0}\right\}\right)$ given by

$$
\mathscr{P}_{R^{-}+T_{j}}=\left\{\operatorname{Conv}\left(\tau_{1} \cup \tau_{2}\right) \mid \tau_{1} \in \mathscr{P}_{1}, \tau_{2} \in \mathscr{P}_{2}\right\}
$$

and then a polyhedral decomposition of $\sigma_{j}^{+}$given by

$$
\mathscr{P}_{\sigma_{j}^{+}}=\left\{\tau_{1}+\tau_{2} \mid \tau_{1} \in \mathscr{P}_{1}, \tau_{2} \in \mathscr{P}_{2}\right\}
$$

The polytopes of $\mathscr{P}_{\sigma_{j}^{+}}$contained in $\partial \sigma_{j}^{+}$are as depicted:

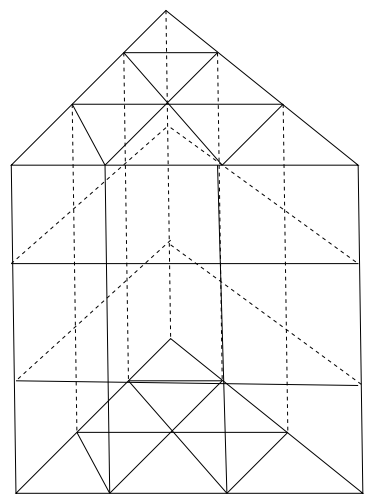

As there is no monodromy around any loop contained in $\operatorname{Int}\left(\sigma_{j}^{+}\right)$, it is enough to focus on the boundary. By applying Proposition 3.15, one finds that the only bit of the discriminant locus that survives is as depicted:

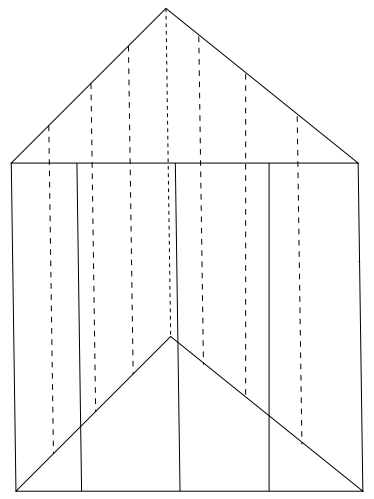

We have a similar picture for $\sigma_{i}^{-}$. 
Finally let, for $i, k, l=0,1,2$,

$$
\begin{aligned}
{ }^{+} \tau_{i}^{k}= & \operatorname{Conv}\left\{\frac{k}{3} P_{i+1}^{+}+\frac{3-k}{3} P_{i+2}^{+}, \frac{k+1}{3} P_{i+1}^{+}+\frac{2-k}{3} P_{i+2}^{+},\right. \\
& \left.\frac{k}{3} P_{i+1}^{0}+\frac{3-k}{3} P_{i+2}^{0}, \frac{k+1}{3} P_{i+1}^{0}+\frac{2-k}{3} P_{i+2}^{0}\right\}, \\
{ }^{-} \tau_{i}^{k}= & \operatorname{Conv}\left\{\frac{k}{3} Q_{i+1}^{-}+\frac{3-k}{3} Q_{i+2}^{-}, \frac{k+1}{3} Q_{i+1}^{-}+\frac{2-k}{3} Q_{i+2}^{-},\right. \\
& \left.\frac{k}{3} Q_{i+1}^{0}+\frac{3-k}{3} Q_{i+2}^{0}, \frac{k+1}{3} Q_{i+1}^{0}+\frac{2-k}{3} Q_{i+2}^{0}\right\}, \\
{ }^{+} \rho_{i}^{l}= & \operatorname{Conv}\left\{\frac{l}{3} Q_{i+1}^{0}+\frac{3-l}{3} Q_{i+2}^{0}, \frac{l+1}{3} Q_{i+1}^{0}+\frac{2-l}{3} Q_{i+2}^{0}\right\}, \\
-\rho_{i}^{l}= & \operatorname{Conv}\left\{\frac{l}{3} P_{i+1}^{0}+\frac{3-l}{3} P_{i+2}^{0}, \frac{l+1}{3} P_{i+1}^{0}+\frac{2-l}{3} P_{i+2}^{0}\right\} .
\end{aligned}
$$

Then for a fixed $i, j$,

$$
\left\{\operatorname{Conv}\left\{{ }^{+} \tau_{i}^{k},{ }^{+} \rho_{j}^{l}\right\} \mid 0 \leq k, l \leq 2\right\} \cup\left\{\operatorname{Conv}\left\{{ }^{-} \rho_{i}^{k},{ }^{-} \tau_{j}^{l}\right\} \mid 0 \leq k, l \leq 2\right\}
$$

form the maximal cells of a polyhedral decomposition of the face of $\nabla^{*}$ dual to the vertex $S_{i}+T_{j}$ of $\nabla$. Then this induces a decomposition of $\sigma_{i j}$ with maximal cells of the form

$$
\left\{{ }^{+} \tau_{i}^{k}+{ }^{+} \rho_{j}^{l} \mid 0 \leq k, l \leq 2\right\} \cup\left\{{ }^{-} \rho_{i}^{k}+{ }^{-} \tau_{j}^{l} \mid 0 \leq k, l \leq 2\right\} .
$$

On the boundary of $\sigma_{i j}$ this looks like

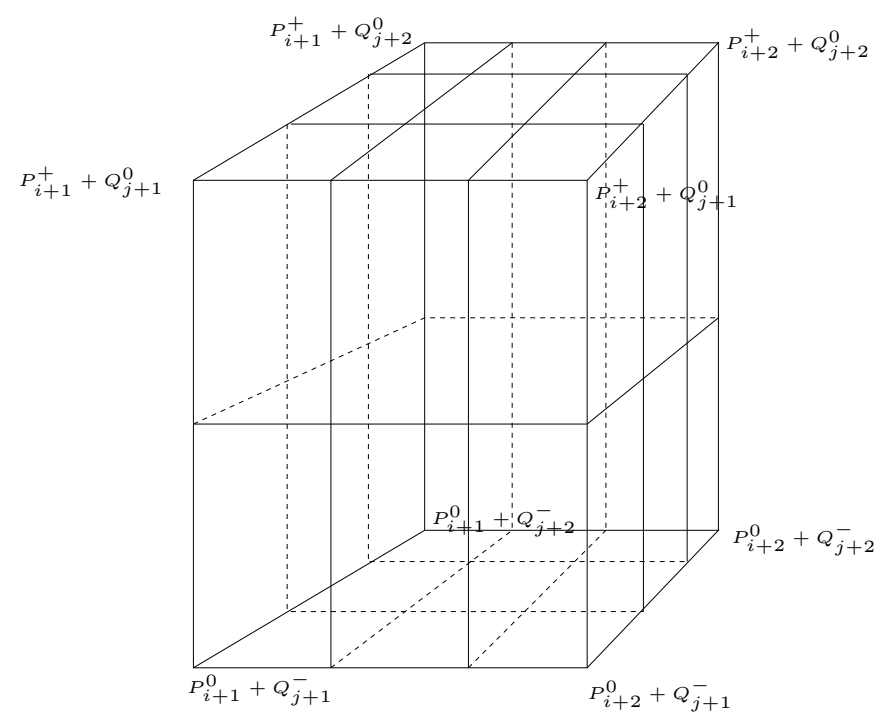

and the discriminant locus on the boundary of $\sigma_{i j}$ looks like 


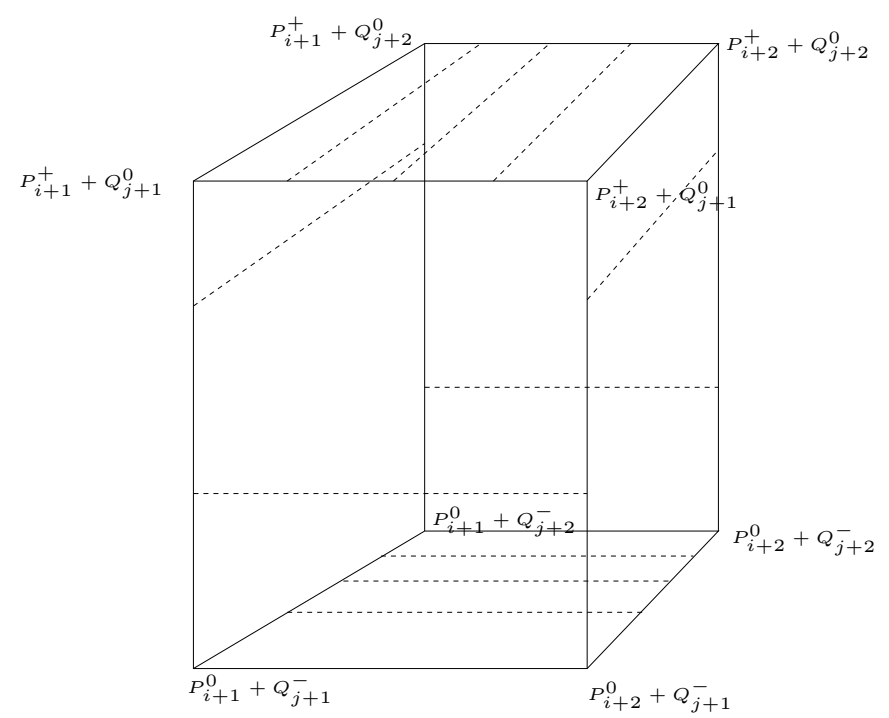

Putting this all together, we can describe the discriminant locus as consisting of 24 circles, occurring in two sets of 12 . As a link, this can be viewed as taking the Hopf link and replacing each circle of the Hopf link with 12 parallel circles.

Now so far we have only followed through the combinatorics, and gotten a specific dual intersection complex. The main point of this section is to observe that this affine manifold, and a more general version of this manifold, can be obtained abstractly using surgeries of affine manifolds, inspired by Kovalev's construction [10]. The starting point is to find a structure of integral affine manifold with singularities with boundary on a closed 2-disk $D$, so that the boundary is an affine line of integral length and such that the affine structure on $D$ has 12 singular points in the interior with monodromy conjugate to $\left(\begin{array}{ll}1 & 1 \\ 0 & 1\end{array}\right)$. We leave as an exercise a construction of an example of such; for a hint, either study carefully the construction above or consult [11]. The boundary of $D$ is an affine manifold isomorphic to $\mathbb{R} / n \mathbb{Z}$ for some integer $n$. Take $B_{1}=B_{2}=D \times(\mathbb{R} / n \mathbb{Z})$, so $\partial B_{1}=\partial B_{2}=(\mathbb{R} / n \mathbb{Z}) \times(\mathbb{R} / n \mathbb{Z})$, and glue $\partial B_{1}$ and $\partial B_{2}$ along their boundaries using an involution $\iota$ of $(\mathbb{R} / n \mathbb{Z}) \times(\mathbb{R} / n \mathbb{Z})$ given by $\iota(a, b)=(b, a)$. After gluing, we get $B$, and because we are gluing $B_{1}$ and $B_{2}$ across an affine plane, we can define an affine structure on $B$ restricting to the given affine structures on $B_{1}$ and $B_{2}$. With the correct choice of $D$, one can show one obtains the same affine manifold which we constructed as a dual intersection complex (where incidentally $n=9)$.

Now the point is that even if we didn't know about the Batyrev-Borisov construction, we can easily produce a degeneration with $B$ as dual intersection complex. Begin by finding a toric degeneration of rational elliptic surfaces (well, we haven't given a definition of such a degeneration, but use your imagination with Definition 1.1 as a guide); in particular, we 
have a diagram

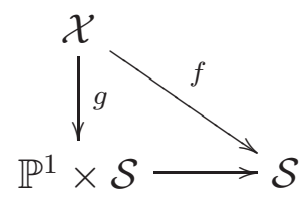

A typical example of such a degeneration is obtained in $\mathbb{P}^{1} \times \mathbb{P}^{2} \times \mathcal{S}$ with equation $t f+x_{0} y_{0} y_{1} y_{2}=0$ where $f$ is a general polynomial of bidegree $(1,3)$ and $\left(x_{0}, x_{1}\right),\left(y_{0}, y_{1}, y_{2}\right)$ are coordinates on $\mathbb{P}^{1}$ and $\mathbb{P}^{2}$ respectively. This is actually not quite general enough (we will obtain a disk $D$ below with, instead of 12 singular points, only three points of multiplicity four), but this should give enough of a hint. As in this example, we will assume $g: \mathcal{X}_{0} \rightarrow \mathbb{P}^{1}$ has as general fibre an $I_{n}$ fibre, and in particular $g^{-1}(\infty)$ is such a fibre, but $g^{-1}(0)$ can be a union of rational surfaces. If we remove $g^{-1}(\{\infty\} \times \mathcal{S})$ from $\mathcal{X}$, we obtain a family of open surfaces, and the dual intersection complex of this family makes sense as a manifold with boundary: the fans of the open components of $\mathcal{X}_{0}$ are not complete, and the corresponding vertices are in the boundary of the dual intersection complex, which will be a disk $D$ with an affine structure with singularities. The boundary of $D$ is $\mathbb{R} / n \mathbb{Z}$.

To construct a degeneration of Schoen's fibred products, blow up the point $\{\infty\} \times\{0\}$ in $\mathbb{P}^{1} \times \mathcal{S}$ and the curve $g^{-1}(\{\infty\} \times\{0\})$ in $\mathcal{X}$ to get

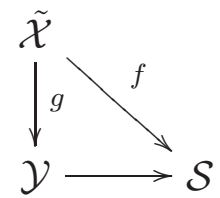

Now take another similar family $\mathcal{X}^{\prime} \rightarrow \mathcal{S}$, in which the role of 0 and $\infty$ in $\mathbb{P}^{1}$ is reversed. We blow up $\{0\} \times\{0\}$ in $\mathbb{P}^{1} \times \mathcal{S}$ this time to get $\mathcal{Y}^{\prime}$, and $\left(g^{\prime}\right)^{-1}(\{0\} \times\{0\})$ in $\mathcal{X}^{\prime}$ to give

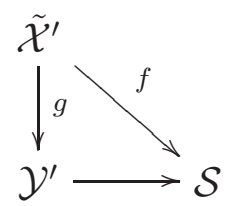

We can identify $\mathcal{Y}$ and $\mathcal{Y}^{\prime}$ so that the exceptional curve on $\mathcal{Y}$ is identified with the proper transform of $\mathbb{P}^{1} \times\{0\}$ on $\mathcal{Y}^{\prime}$, and vice versa. We then take $\mathcal{Z}=\tilde{\mathcal{X}} \times \mathcal{Y} \tilde{\mathcal{X}}^{\prime} \rightarrow \mathcal{S}$ as a toric degeneration of Schoen's fibred product. It is easy to see the dual intersection complex is constructed by gluing two copies of $D \times S^{1}$ with the twist as described above.

The decomposition of $B$ into $B_{1}$ and $B_{2}$ actually corresponds, in some sense, to a simpler degeneration of the Schoen threefolds into a union of two threefolds, essentially as considered in [10], on which one can explicitly find special Lagrangian fibrations. In our algebro-geometric picture, we degenerate these two threefolds further, to the threefolds sitting over the two curves in $\mathcal{Y}$ sitting over $0 \in \mathcal{S}$. As a last observation, we note that it is easy to see that dualizing the torus fibration obtained from $B$ doesn't change the 
topology, using the fact that two-dimensional torus bundles are self-dual. Hence we see an explicit prediction of the Strominger-Yau-Zaslow approach that the Schoen fibred product is self-mirror, something which may not have been self-evident from the Batyrev-Borisov picture.

\section{REFERENCES}

[1] Borisov, L., "Towards the Mirror Symmetry for Calabi-Yau Complete intersections in Gorenstein Toric Fano Varieties," preprint (1993), alg-geom/9310001.

[2] Batyrev, V., and Borisov, L., "On Calabi-Yau complete intersections in toric varieties," in Higherdimensional complex varieties (Trento, 1994), 39-65, de Gruyter, Berlin, 1996.

[3] Gross, M., and Siebert, B., "Affine manifolds, log structures, and mirror symmetry," Turkish J. Math., 27, (2003), 33-60.

[4] Gross, M., and Siebert, B., "Mirror Symmetry via Logarithmic Degeneration Data I," preprint (2003), math.AG/0309070, Third Version.

[5] Gross, M., and Siebert, B., "Torus fibrations and toric degenerations," in preparation.

[6] Haase, C., and Zharkov, I., "Integral affine structures on spheres and torus fibrations of Calabi-Yau toric hypersurfaces I," preprint, 2002, math.AG/0205321.

[7] Haase, C., and Zharkov, I., "Integral affine structures on spheres III: complete intersections," preprint, 2005, math.AG/0504181.

[8] Hosono, S., Saito, M-H., Stienstra, J., "On the mirror symmetry conjecture for Schoen's CalabiYau 3-folds," in Integrable systems and algebraic geometry (Kobe/Kyoto, 1997), 194-235, World Sci. Publishing, River Edge, NJ, 1998.

[9] Hu, S., "Semi-stable Degeneration of Toric Varieties and their hypersurfaces," preprint (2001), math.AG/0110091.

[10] Kovalev, A., IPAM lecture, April 2003.

[11] Leung, N.-C, Symington, M., "Almost toric symplectic four-manifolds," preprint, 2003, math.SG/0312165.

[12] Matsumura, H., Commutative algebra. Second edition. Mathematics Lecture Note Series, 56. Benjamin/Cummings Publishing Co., Inc., Reading, Mass., 1980.

[13] Oda, T., Convex bodies and algebraic geometry. An introduction to the theory of toric varieties, Ergebnisse der Mathematik und ihrer Grenzgebiete (3), 15. Springer-Verlag, Berlin, 1988.

[14] Ruan, W.-D., "Lagrangian torus fibration and mirror symmetry of Calabi-Yau hypersurface in toric variety," preprint, 2000, math.DG/0007028.

[15] Schoen, C., "On fiber products of rational elliptic surfaces with section," Math. Z. 197 (1988), 177-199.

[16] Strominger, A., Yau, S.-T., and Zaslow, E., "Mirror Symmetry is T-Duality," Nucl. Phys. B479, (1996) 243-259.

[17] Zharkov, I., "Torus fibrations of Calabi-Yau hypersurfaces in toric varieties," Duke Math. J. 101 (2000), 237-257.

Department of Mathematics, UCSD, 9500 Gilman Drive, La Jolla, CA 92093-0112, USA

E-mail address: mgross@math.ucsd.edu 